\title{
MID-INFRARED PROPERTIES OF THE SWIFT BURST ALERT TELESCOPE ACTIVE GALACTIC NUCLEI SAMPLE OF THE LOCAL UNIVERSE. I. EMISSION-LINE DIAGNOSTICS
}

\author{
K. A. Weaver ${ }^{1}$, M. Meléndez ${ }^{1,5,6}$, R. F. Mushotzky ${ }^{1,7}$, S. Kraemer ${ }^{1,8}$, K. Engle $^{1,9}$, E. Malumuth ${ }^{1,10}$, J. Tueller ${ }^{1}$, \\ C. Markwardt ${ }^{1,7}$, C. T. Berghea ${ }^{2,11}$, R. P. Dudik ${ }^{2}$, L. M. Winter ${ }^{3,12}$, And L. Armus ${ }^{4}$ \\ ${ }^{1}$ NASA Goddard Space Flight Center, Greenbelt, MD, 20771, USA \\ ${ }^{2}$ U.S. Naval Observatory, Washington, DC 20392, USA \\ ${ }^{3}$ Center for Astrophysics and Space Astronomy, University of Colorado, Boulder, CO 80309-0440, USA \\ ${ }^{4}$ Spitzer Science Center, California Institute of Technology, Pasadena, CA 91125, USA \\ Received 2009 November 9; accepted 2010 April 29; published 2010 May 27
}

\begin{abstract}
We compare mid-infrared emission-line properties from high-resolution Spitzer spectra of a hard X-ray $(14-195 \mathrm{keV})$ selected sample of nearby $(z<0.05)$ active galactic nuclei (AGNs) detected by the Burst Alert Telescope (BAT) aboard Swift. The luminosity distribution for the mid-infrared emission lines, [O IV] $25.89 \mu \mathrm{m}$, [Ne II] $12.81 \mu \mathrm{m}$, [Ne III] $15.56 \mu \mathrm{m}$, and [Ne v] 14.32/24.32 $\mu \mathrm{m}$, and hard X-ray continuum show no differences between Seyfert 1 and Seyfert 2 populations; however, six newly discovered BAT AGNs are under-luminous in [O IV], most likely the result of dust extinction in the host galaxy. The overall tightness of the mid-infrared correlations and BAT fluxes and luminosities suggests that the emission lines primarily arise in gas ionized by the AGNs. We also compare the mid-infrared emission lines in the BAT AGNs with those from published studies of ULIRGs, Palomar-Green quasars, star-forming galaxies, and LINERs. We find that the BAT AGN sample falls into a distinctive region when comparing the $[\mathrm{Ne}$ III]/[Ne II] and the $[\mathrm{O}$ IV $] /[\mathrm{Ne}$ III] ratios. These line ratios are lower in sources that have been previously classified in the mid-infrared/optical as AGNs than those found for the BAT AGNs, suggesting that, in our X-ray selected sample, the AGNs represent the main contribution to the observed line emission. These ratios represent a new emission line diagnostic for distinguishing between AGNs and star-forming galaxies.
\end{abstract}

Key words: galaxies: active - galaxies: Seyfert - infrared: galaxies - X-rays: galaxies

Online-only material: color figures

\section{INTRODUCTION}

Active galactic nuclei (AGNs) span over 7 orders of magnitude in bolometric luminosity $\left(L_{\mathrm{bol}} ;\right.$ Koratkar \& Blaes 1999$)$ and yet are all believed to be powered by the same physical mechanism: accretion of matter onto supermassive black holes (e.g., Rees 1984; Peterson et al. 2004). One way to approach the study of AGNs is to concentrate on those in the local universe (e.g., $z<0.05$ ), which permits us, among other things, to determine the properties of the host galaxy. Such studies tend to focus on Seyfert galaxies, which are modest luminosity AGNs $\left(L_{\text {bol }} \lesssim 10^{45} \mathrm{erg} \mathrm{s}^{-1}\right)$, but bright enough, due to their proximity, to be studied across the full electromagnetic spectrum.

Although Seyfert galaxies and other AGNs have been traditionally defined in terms of their optical properties (e.g., classification into Type I and Type II; Khachikian \& Weedman 1974), sample selection of AGNs via a single waveband can lead to observational bias (e.g., Mulchaey et al. 1994). For example, most AGNs are obscured from our line of sight by dust and gas (Matt 2000) and any selection based on optical (or UV) properties would miss many objects or could highly skew a sample toward unobscured objects (e.g., Barger et al. 2005). The soft $\mathrm{X}$-ray properties of Seyfert galaxies generally follow the same

\footnotetext{
5 NASA Postdoctoral Program Fellow.

6 Current address: Department of Physics and Astronomy, Johns Hopkins University, Baltimore, MD 21218, USA.

7 University of Maryland, College Park, MD 20742, USA.

8 Institute for Astrophysics and Computational Sciences, Department of Physics, The Catholic University of America, Washington, DC 20064, USA.

9 Adnet.

10 SESDA.

${ }^{11}$ Computational Physics, Inc., Sprinfield, VA 22151, USA.

12 Hubble Fellow.
}

dichotomy as their optical properties. The X-ray continuum source in Seyfert 1s can be observed directly (e.g., George et al. 1998), while the central X-ray source is sometimes undetectable in Seyfert $2 \mathrm{~s}$, due to material with $N_{\mathrm{H}}>10^{22.5} \mathrm{~cm}^{-2}$ along our line of sight. There is additionally a wide range of effective IR colors (Kuraszkiewicz et al. 2003; Lutz et al. 2005) which can introduce selection bias. A comparison of infrared and X-ray data (Franceschini et al. 2005) shows a factor of 30 range in the IR $24 \mu \mathrm{m}$ to $\sim 4 \mathrm{keV} \mathrm{X-ray} \mathrm{flux} \mathrm{ratio} \mathrm{for} \mathrm{X-ray} \mathrm{selected}$ AGNs, suggesting a range of geometries and optical depths for dust reprocessing, and probably variance in the intrinsic powerlaw AGN continuum (see McKernan et al. 2009 for discussion). Obscuration and star formation in the host galaxy can also dominate and introduce confusion in the IR (e.g., Lutz et al. 2004; Barmby et al. 2006). In fact, virtually all surveys for AGNs based purely on IR, optical, UV, or soft X-ray data have been biased (Mushotzky 2004). Even Sloan surveys (Heckman et al. 2004) or IR surveys (Franceschini et al. 2005) have required indirect AGN indicators which are known to be not necessarily robust (Meléndez et al. 2008b).

To understand the intrinsic properties of AGNs as a class, it is critical to start with a survey where we can be as certain as possible that we are viewing the AGN-only parts of these galaxies. At X-ray energies of $E>10-20 \mathrm{keV}$, the obscuring material is relatively optically thin for column densities less than $\sim 3 \times 10^{24} \mathrm{~cm}^{-2}$ (Compton-thin objects). Even if an AGN is well buried within its host galaxy there is an unaffected view of the central power source. A hard X-ray survey should thus find all Compton thin AGNs in a uniform fashion and is the most representative, since at present, there are very few, if any, known X-ray "quiet" AGNs. Such a hard X-ray survey is now available from the Swift Burst Alert Telescope (BAT). The Swift 
BAT is sensitive over $\sim 85 \%$ of the sky to a flux threshold of $2 \times 10^{-11} \mathrm{erg} \mathrm{cm}^{-2} \mathrm{~s}^{-1}$ in the $14-195 \mathrm{keV}$ band (Markwardt et al. 2005). The BAT data are about 10 times more sensitive than the previous hard X-ray all sky survey (Levine et al. 1984). The BAT detects all bright AGNs, whether they are obscured or not. Moreover, several of the BAT sources are newly discovered AGNs or have been poorly studied, if at all, at other wavelengths (Winter et al. 2008; Tueller et al. 2008, 2010).

Nevertheless, although all of the BAT-detected objects are true AGNs, in order to fully explore the properties of these AGNs one needs to take a multi-wavelength approach. For example, studying the IR properties of the BAT AGNs will provide insight into the IR/X-ray scatter and thus determine the true distribution of IR properties. There have been a large number of studies of the mid-infrared emission line properties of active galaxies using both Infrared Space Observatory (Kessler et al. 1996) and Spitzer Space Telescope (Werner et al. 2004). The ratios of high- and low-ionization mid-infrared emission lines have been widely used to separate the relative contribution of the AGNs and star formation (e.g., Genzel et al. 1998; Sturm et al. 2002; Dale et al. 2006; Armus et al. 2007; Farrah et al. 2007; Meléndez et al. 2008a; Baum et al. 2010). More recently, (Hao et al. 2009, H09) used new high-resolution Spitzer spectroscopy to probe the utility of mid-infrared emission line diagnostics as a way to separate active galaxies from star-forming galaxies. In our first study of mid-infrared properties of the BAT AGNs (Meléndez et al. 2008b), we found the [O IV]25.89 $\mu \mathrm{m}$ to be an accurate indicator of the AGN luminosity, with an uncertainty of $\sim 0.3 \mathrm{dex}$; this result has been confirmed using larger samples (Rigby et al. 2009; Diamond-Stanic et al. 2009). Using a complete, volume-limited, sample of galaxies (Goulding \& Alexander 2009, GA09) demonstrated the utility of highionization mid-infrared emission lines, such as [Ne v $] 14.32 \mu \mathrm{m}$, to identify AGNs including those that were not identified as AGNs in optical studies (see also, Landi et al. 2007; Abel \& Satyapal 2008; Dudik et al. 2009; Satyapal et al. 2009). Similar results have been found by (Bernard-Salas et al. 2009, B09) in their study of starburst galaxies.

This paper is the first in a series seeking to understand the nature of the observed mid-infrared luminosities in AGNs and their wide variety of spectral forms. Here we report results from the portion of our sample that have high-resolution Spitzer spectra. This work complements the extensive optical imaging and spectroscopy of the AGN population (Koss et al. 2010; Winter et al. 2010) and the detailed analysis of the X-ray properties of the BAT AGN sample (e.g., Winter et al. 2008, 2009a, 2009b). In following papers, we will report on the results from our analysis of the low-resolution Spitzer spectra which will include the study of polycyclic aromatic hydrocarbon features, silicate absorption, and mid-infrared continuum properties of the BAT AGN sample. In order to calculate the luminosities presented in this work we assumed a flat universe with a Hubble constant $H_{o}=71 \mathrm{~km} \mathrm{~s}^{-1} \mathrm{Mpc}^{-1}, \Omega_{\Lambda}=0.73$, and $\Omega_{\mathrm{M}}=0.27$, with redshift values taken from NASA's ExtraGalactic Database (NED), except for sources with redshift values of $z<0.01$ where distances are taken from The Extragalactic Distance Database (EDD; Tully 1988; Tully et al. 2009).

\section{OBSERVATIONS AND ANALYSIS}

\subsection{X-ray Data and AGN Sample Selection}

Our sample was selected from the first unbiased local AGN sample obtained with the Swift BAT survey. The X-ray positions have $\sim 3^{\prime \prime}$ positional uncertainties and we are highly confident of the positions and the optical identifications. Source identifications are based primarily on the X-ray imaging data and a correlation with optical images and catalogs. In some cases, the identifications are based on positional coincidences with previously known AGNs.

The median redshift of the BAT objects is $z \sim 0.025$. Our selection criteria are $z<0.05,|b|>19^{\circ}$ and a hard X-ray BAT flux of $>2 \times 10^{-11} \mathrm{erg} \mathrm{cm}^{-2} \mathrm{~s}^{-1}$. The flux limit provides sufficient $\mathrm{S} / \mathrm{N}$ to be sure that the sources are statistically robust. Our total BAT sample contains 130 objects above a significance threshold of $5.0 \sigma$. We obtained from the Infrared Spectrograph (IRS; Houck et al. 2004) on board Spitzer high- and lowresolution spectra for 60 of these objects, while 70 previously had Spitzer IRS spectra from other observing programs. There are published BAT AGN catalogs from two surveys, the 9 month survey (Tueller et al. 2008) and an updated 22 month catalog (Tueller et al. 2010). Here we use the 22 month fluxes from Tueller et al. (2010) as the 22 month survey is the most sensitive. However, in our sample, four of the AGNs in the 9 month survey were not detected in the 22 month survey; therefore, we will use the fluxes from the 9 month survey as upper limits (see footnote in Table 1).

\subsection{Spitzer Observations and Data Analysis}

In this work, we discuss only the high-resolution Spitzer spectroscopy of our total sample, which result on a subsample of 79 BAT AGNs. The galaxies in our sample were observed with IRS in the short-high $\left(\mathrm{SH}, \lambda=9.9-19.6 \mu \mathrm{m}, 4\right.$. $7 \times 11^{\prime \prime} .3$, $R \sim 600)$ and long-high (LH, $\lambda=18.7-37.2 \mu \mathrm{m}, 11^{\prime \prime} .1 \times 22^{\prime \prime} .3$, $R \sim 600)$ IRS order in the staring mode. Science targets in the staring mode are placed at two node position along the IRS slit. This sample includes fluxes found in the literature (Weedman et al. 2005; Armus et al. 2006; Tommasin et al. 2008) and from our analysis of unpublished archival spectra observed with IRS, including observations collected with the Spitzer Cycle 3 and Cycle 5 GTO program "Spitzer Observations to Complete the First Unbiased AGN Sample of the Local Universe" (P30745 and P50588, PI: K. Weaver). This high-resolution sample includes 38 Seyfert 1s, 33 Seyfert 2s, six previously unknown or poorly studied AGNs and two LINERs. High-resolution spectroscopy allows us to easily separate blended features such as [O IV] $-[\mathrm{Fe}$ II] $25.99 \mu \mathrm{m}$ and [Ne v] $14.32 \mu \mathrm{m}-[\mathrm{Cl} \mathrm{II}]$ $14.37 \mu \mathrm{m}$. Moreover, the subsample presented in this work spans the same range on $14-195 \mathrm{keV}$ luminosities as the 22 month BAT AGN sample and is large enough to be statistically representative of the whole sample of 130 AGNs. Furthermore, the Kolmogorov-Smirnov (K-S) test probabilities for Seyfert 1 and Seyfert 2 galaxies between the subsample presented in this work and the whole sample of 130 sources are $46.2 \%$ and $11.5 \%,{ }^{13}$ respectively, meaning that there are no differences in their X-ray luminosities. Finally, our sample also has the same range in mid-infrared emission line luminosities as complete samples such as the $12 \mu \mathrm{m}$ (Tommasin et al. 2010) and the revised Shapley-Ames sample (Rigby et al. 2009).

For the analysis of Spitzer data we used the basic calibrated data files preprocessed using the S17.2 IRS pipeline. This includes ramp fitting, dark sky subtraction, drop correction,

\footnotetext{
13 A probability value of less than 5\% represents a high level of significance that two samples drawn from the same parent population would differ this much $5 \%$ of the time, i.e., that they are different. A strong level of significance is obtained for values smaller than 1\% (e.g., Press et al. 1992; Bevington \& Robinson 2003)
} 
Table 1

Spitzer IRS High-resolution Spectroscopy of the BAT Sample of AGNs

\begin{tabular}{|c|c|c|c|c|c|c|c|c|}
\hline Name & $\begin{array}{c}\text { Distance } \\
(\mathrm{Mpc})\end{array}$ & Type $^{\mathrm{a}}$ & $\mathrm{BAT}^{\mathrm{b}}$ & $\begin{array}{c}{[\mathrm{Ne} \mathrm{II}]} \\
(12.81 \mu \mathrm{m})\end{array}$ & $\begin{array}{c}{[\mathrm{Ne} v]} \\
(14.32 \mu \mathrm{m})\end{array}$ & $\begin{array}{c}{[\mathrm{Ne} \text { III] }} \\
(15.56 \mu \mathrm{m})\end{array}$ & $\begin{array}{c}{[\mathrm{Ne} v]} \\
(24.32 \mu \mathrm{m})\end{array}$ & $\begin{array}{c}{[\mathrm{O} \text { IV }]} \\
(25.89 \mu \mathrm{m}) \\
\end{array}$ \\
\hline & & & & \multicolumn{5}{|c|}{ Integrated Line Fluxes $\left(10^{-21} \mathrm{~W} \mathrm{~cm}^{-2}\right)$} \\
\hline 2MASX J05580206-3820043 & 146.8 & 1 & $3.99 \pm 0.37$ & $3.20 \pm 0.89$ & $1.89 \pm 0.13$ & $4.72 \pm 0.14$ & $3.80 \pm 0.51$ & $3.69 \pm 0.45$ \\
\hline $3 \mathrm{C} 120$ & 143.0 & 1 & $11.89 \pm 0.63$ & $9.19 \pm 0.66$ & $17.21 \pm 0.28$ & $27.43 \pm 0.92$ & $27.63 \pm 8.92$ & $116.68 \pm 1.22$ \\
\hline Ark 120 & 141.7 & 1 & $7.08 \pm 0.57$ & $3.47 \pm 0.91$ & $<1.18$ & $4.33 \pm 0.79$ & $<1.29$ & $4.03 \pm 0.38$ \\
\hline Cen $A^{c, d}$ & 4.9 & 2 & $92.62 \pm 0.71$ & 193.00 & 23.16 & 147.65 & 29.92 & 131.24 \\
\hline ESO 005-G004 ${ }^{\mathrm{d}}$ & 22.4 & .. & $4.48 \pm 0.60$ & $16.64 \pm 1.19$ & $0.50 \pm 0.12$ & $5.29 \pm 0.34$ & $<0.49$ & $4.49 \pm 0.19$ \\
\hline ESO 033- G002 & 77.5 & 2 & $2.61 \pm 0.45$ & $2.67 \pm 0.18$ & $5.57 \pm 0.56$ & $8.34 \pm 0.56$ & $5.37 \pm 0.51$ & $13.85 \pm 0.73$ \\
\hline ESO 103-G035 & 56.7 & 2 & $11.14 \pm 0.59$ & $30.92 \pm 2.07$ & $15.85 \pm 3.49$ & $41.62 \pm 1.78$ & $10.31 \pm 0.87$ & $34.40 \pm 0.75$ \\
\hline ESO 140-G043 & 60.5 & 1 & $4.57 \pm 0.66$ & $11.41 \pm 0.26$ & $7.83 \pm 0.70$ & $13.93 \pm 0.32$ & $7.95 \pm 0.44$ & $27.46 \pm 0.35$ \\
\hline ESO 323-G077 & 64.2 & 1.2 & $4.70 \pm 0.66$ & $40.16 \pm 1.41$ & $5.44 \pm 1.61$ & $18.07 \pm 1.07$ & $6.19 \pm 0.14$ & $25.11 \pm 0.62$ \\
\hline ESO 362-G018 & 53.1 & 1.5 & $6.22 \pm 0.52$ & $9.94 \pm 0.23$ & $3.15 \pm 0.37$ & $7.16 \pm 0.18$ & $2.75 \pm 0.20$ & $10.11 \pm 0.37$ \\
\hline ESO 417- G006 & 69.7 & 2 & $3.06 \pm 0.46$ & $1.77 \pm 0.14$ & $0.42 \pm 0.10$ & $2.54 \pm 0.07$ & $0.47 \pm 0.16$ & $4.04 \pm 0.18$ \\
\hline F9 & 205.9 & 1 & $5.07 \pm 0.45$ & $2.91 \pm 0.68$ & $<2.66$ & $5.31 \pm 0.37$ & $2.74 \pm 0.37$ & $6.08 \pm 0.26$ \\
\hline F49 & 86.2 & 2 & $2.93 \pm 0.54$ & $39.55 \pm 2.93$ & $25.50 \pm 2.96$ & $47.12 \pm 2.43$ & $12.74 \pm 0.36$ & $39.22 \pm 2.37$ \\
\hline IC 486 & 114.8 & 1 & $3.22 \pm 0.70$ & $6.86 \pm 0.05$ & $3.30 \pm 0.20$ & $6.79 \pm 0.21$ & $2.84 \pm 0.01$ & $11.19 \pm 0.29$ \\
\hline IC 1816 & 72.5 & 1 & $2.58 \pm 0.48$ & $18.36 \pm 1.51$ & $6.21 \pm 0.61$ & $19.64 \pm 1.17$ & $4.34 \pm 0.30$ & $16.52 \pm 1.81$ \\
\hline IC 4329A & 68.7 & 1.2 & $33.08 \pm 0.62$ & $30.19 \pm 2.76$ & $32.79 \pm 6.01$ & $65.30 \pm 0.95$ & $29.90 \pm 1.74$ & $103.58 \pm 3.24$ \\
\hline IC 5063 & 48.3 & 2 & $8.59 \pm 0.72$ & $28.22 \pm 3.34$ & $35.97 \pm 1.58$ & $73.67 \pm 4.61$ & $25.86 \pm 1.20$ & $117.21 \pm 11.13$ \\
\hline MCG-01-13-025 & 68.0 & 1.2 & $<4.5$ & $2.87 \pm 0.18$ & $<0.18$ & $1.90 \pm 0.05$ & $<1.44$ & $0.76 \pm 0.18$ \\
\hline MCG-05-23-016 & 36.1 & 2 & $20.77 \pm 0.56$ & $18.13 \pm 0.42$ & $11.06 \pm 0.50$ & $16.96 \pm 0.93$ & $11.19 \pm 0.26$ & $27.95 \pm 7.61$ \\
\hline MCG-01-24-012 & 84.2 & 2 & $4.58 \pm 0.51$ & $6.74 \pm 0.41$ & $2.90 \pm 0.27$ & $6.07 \pm 0.36$ & $2.30 \pm 0.58$ & $10.07 \pm 0.77$ \\
\hline MCG-02-58-22 & 205.1 & 1.5 & $10.17 \pm 0.57$ & $5.29 \pm 0.39$ & $2.80 \pm 0.34$ & $9.71 \pm 0.16$ & $3.05 \pm 0.39$ & $13.74 \pm 1.74$ \\
\hline MCG-03-34-064 & 70.8 & 1.8 & $3.15 \pm 0.45$ & $57.83 \pm 7.29$ & $54.98 \pm 5.26$ & $120.13 \pm 6.88$ & $35.46 \pm 1.92$ & $110.83 \pm 9.35$ \\
\hline MCG-06-30-015 & 32.9 & 1.2 & $7.82 \pm 0.57$ & $4.20 \pm 0.12$ & $6.05 \pm 0.76$ & $6.62 \pm 0.33$ & $6.62 \pm 0.05$ & $23.49 \pm 0.81$ \\
\hline $\operatorname{MRK} 3^{\mathrm{c}}$ & 57.7 & 2 & $15.65 \pm 0.61$ & 100.00 & 64.50 & 179.00 & 67.50 & 214.00 \\
\hline MRK $6^{\mathrm{f}}$ & 80.6 & 1.5 & $7.61 \pm 0.55$ & $28.00 \pm 0.23$ & $9.39 \pm 0.19$ & $49.34 \pm 0.32$ & $10.43 \pm 0.21$ & $48.24 \pm 0.27$ \\
\hline MRK $18^{\mathrm{e}}$ & 47.2 & $\ldots$ & $<3.1$ & $17.04 \pm 0.71$ & $0.78 \pm 0.01$ & $8.24 \pm 0.24$ & $<2.25$ & $2.69 \pm 0.14$ \\
\hline MRK 79 & 95.3 & 1.2 & $4.89 \pm 0.52$ & $11.29 \pm 3.71$ & $9.62 \pm 1.07$ & $20.43 \pm 0.50$ & $13.16 \pm 1.10$ & $39.99 \pm 2.68$ \\
\hline MRK 335 & 111.1 & 1.2 & $2.47 \pm 0.41$ & $2.10 \pm 0.31$ & $1.31 \pm 0.11$ & $2.70 \pm 0.15$ & $<1.55$ & $6.31 \pm 0.17$ \\
\hline MRK 348 & 64.2 & 2 & $13.66 \pm 0.56$ & $15.34 \pm 0.74$ & $6.90 \pm 0.36$ & $20.60 \pm 0.79$ & $4.75 \pm 0.27$ & $17.87 \pm 0.23$ \\
\hline MRK 352 & 63.5 & 1 & $4.16 \pm 0.50$ & $0.54 \pm 0.08$ & $<0.16$ & $0.34 \pm 0.02$ & $<0.23$ & $0.26 \pm 0.04$ \\
\hline MRK 509 & 149.2 & 1.2 & $9.44 \pm 0.68$ & $11.98 \pm 1.06$ & $7.61 \pm 1.74$ & $17.32 \pm 3.29$ & $7.60 \pm 0.10$ & $27.54 \pm 0.42$ \\
\hline MRK $590^{\mathrm{e}}$ & 112.7 & 1.2 & $<3.7$ & $5.26 \pm 0.46$ & $<3.22$ & $5.42 \pm 0.12$ & $<1.23$ & $1.79 \pm 0.26$ \\
\hline MRK 766 & 55.2 & 1.5 & $2.42 \pm 0.29$ & $24.27 \pm 0.94$ & $22.14 \pm 0.23$ & $23.52 \pm 1.41$ & $18.32 \pm 1.60$ & $46.47 \pm 0.84$ \\
\hline MRK 817 & 136.1 & 1.5 & $2.21 \pm 0.36$ & $4.57 \pm 0.88$ & $2.88 \pm 0.48$ & $5.51 \pm 0.77$ & $4.22 \pm 0.73$ & $6.06 \pm 0.23$ \\
\hline MRK 841 & 158.2 & 1.5 & $2.93 \pm 0.37$ & $3.18 \pm 0.18$ & $8.12 \pm 0.31$ & $12.46 \pm 0.73$ & $6.96 \pm 0.33$ & $22.91 \pm 1.08$ \\
\hline NGC $454^{\mathrm{e}}$ & 51.8 & 2 & $<2.3$ & $4.70 \pm 0.40$ & $3.73 \pm 0.14$ & $6.40 \pm 0.17$ & $5.54 \pm 0.31$ & $15.80 \pm 0.85$ \\
\hline NGC 513 & 83.8 & 2 & $2.06 \pm 0.43$ & $10.38 \pm 1.78$ & $1.52 \pm 0.23$ & $4.71 \pm 0.32$ & $1.38 \pm 0.21$ & $6.69 \pm 0.41$ \\
\hline NGC 788 & 58.1 & 2 & $9.33 \pm 0.57$ & $6.59 \pm 0.11$ & $5.35 \pm 0.34$ & $13.60 \pm 0.50$ & $8.68 \pm 1.28$ & $24.14 \pm 0.36$ \\
\hline NGC 931 & 71.2 & 1.5 & $6.56 \pm 0.50$ & $5.50 \pm 0.59$ & $9.91 \pm 2.31$ & $15.94 \pm 0.52$ & $13.52 \pm 0.58$ & $42.90 \pm 0.63$ \\
\hline NGC 973 & 69.3 & $\ldots$ & $3.09 \pm 0.58$ & $8.03 \pm 0.09$ & $2.07 \pm 0.18$ & $8.92 \pm 0.38$ & $3.21 \pm 0.27$ & $14.11 \pm 2.64$ \\
\hline NGC $1052^{\mathrm{d}}$ & 17.8 & LINER & $3.75 \pm 0.67$ & $22.22 \pm 0.94$ & $0.64 \pm 0.03$ & $12.31 \pm 0.48$ & $2.69 \pm 1.33$ & $2.37 \pm 0.10$ \\
\hline NGC 1194 & 58.0 & 1.9 & $3.64 \pm 0.60$ & $3.27 \pm 0.34$ & $4.48 \pm 0.12$ & $8.27 \pm 0.41$ & $3.98 \pm 0.32$ & $14.39 \pm 0.10$ \\
\hline NGC $1365^{\mathrm{d}}$ & 18.9 & 1.8 & $7.19 \pm 0.44$ & $161.67 \pm 17.48$ & $22.35 \pm 1.97$ & $61.06 \pm 0.90$ & $38.53 \pm 1.50$ & $145.38 \pm 8.89$ \\
\hline NGC 2110 & 33.1 & 2 & $35.01 \pm 0.70$ & $60.19 \pm 5.34$ & $5.22 \pm 0.82$ & $47.40 \pm 0.71$ & $7.65 \pm 0.63$ & $45.71 \pm 3.41$ \\
\hline NGC $2992^{\mathrm{d}}$ & 30.5 & 2 & $4.82 \pm 0.63$ & $53.65 \pm 3.66$ & $32.62 \pm 5.38$ & $61.06 \pm 1.98$ & $27.81 \pm 0.20$ & $114.22 \pm 6.44$ \\
\hline NGC $3079^{\mathrm{c}, \mathrm{d}}$ & 20.4 & 2 & $3.44 \pm 0.44$ & 104.00 & 1.04 & 22.88 & $<1.56$ & 9.26 \\
\hline NGC $3081^{\mathrm{d}}$ & 32.5 & 2 & $10.24 \pm 0.67$ & $12.62 \pm 1.16$ & $12.62 \pm 0.81$ & $36.46 \pm 1.25$ & $35.79 \pm 0.05$ & $119.73 \pm 8.42$ \\
\hline NGC $3227^{\mathrm{d}}$ & 20.6 & 1.5 & $14.13 \pm 0.50$ & $65.05 \pm 6.91$ & $26.23 \pm 1.23$ & $74.62 \pm 2.00$ & $17.82 \pm 0.99$ & $64.91 \pm 2.31$ \\
\hline NGC 3281 & 45.5 & 2 & $9.01 \pm 0.66$ & $19.94 \pm 2.16$ & $47.51 \pm 2.75$ & $58.35 \pm 2.48$ & $42.15 \pm 3.24$ & $174.65 \pm 13.25$ \\
\hline NGC $3516^{\mathrm{d}, \mathrm{f}}$ & 38.9 & 1.5 & $12.54 \pm 0.45$ & $8.07 \pm 0.25$ & $7.88 \pm 0.50$ & $17.72 \pm 0.33$ & $10.39 \pm 0.33$ & $46.92 \pm 0.35$ \\
\hline NGC $3783^{d}$ & 38.5 & 1 & $19.45 \pm 0.66$ & $19.82 \pm 0.79$ & $19.54 \pm 3.44$ & $26.18 \pm 0.57$ & $13.82 \pm 0.29$ & $39.25 \pm 0.07$ \\
\hline NGC $4051^{\mathrm{d}}$ & 17.1 & 1.5 & $4.34 \pm 0.35$ & $19.69 \pm 0.92$ & $10.17 \pm 0.65$ & $16.35 \pm 0.44$ & $16.19 \pm 2.53$ & $36.95 \pm 2.05$ \\
\hline $\mathrm{NGC} 4102^{\mathrm{d}}$ & 17.0 & LINER & $2.58 \pm 0.38$ & $349.81 \pm 39.88$ & $<8.10$ & $30.44 \pm 1.12$ & $<6.29$ & $20.66 \pm 7.69$ \\
\hline NGC $4138^{\mathrm{d}}$ & 17.0 & 1.9 & $3.69 \pm 0.45$ & $3.23 \pm 0.52$ & $0.54 \pm 0.10$ & $2.82 \pm 0.37$ & $<0.83$ & $2.03 \pm 0.18$ \\
\hline NGC $4151^{\mathrm{c}, \mathrm{d}}$ & 20.3 & 1.5 & $62.23 \pm 0.46$ & 134.00 & 77.72 & 204.35 & 67.67 & 236.51 \\
\hline NGC $4235^{d}$ & 35.1 & 1 & $2.40 \pm 0.55$ & $3.69 \pm 0.65$ & $<0.58$ & $3.70 \pm 0.54$ & $0.92 \pm 0.19$ & $3.77 \pm 0.77$ \\
\hline NGC $4388^{d}$ & 16.8 & 2 & $34.64 \pm 0.52$ & $79.74 \pm 4.76$ & $45.35 \pm 0.84$ & $108.18 \pm 1.56$ & $64.10 \pm 0.12$ & $311.42 \pm 25.79$ \\
\hline $\mathrm{NGC} 4395^{\mathrm{d}}$ & 3.9 & 1.8 & $3.12 \pm 0.41$ & $4.74 \pm 0.25$ & $1.47 \pm 0.13$ & $7.02 \pm 0.58$ & $1.49 \pm 0.62$ & $8.16 \pm 0.26$ \\
\hline NGC 4507 & 50.3 & 2 & $22.51 \pm 0.68$ & $33.73 \pm 2.63$ & $12.50 \pm 1.39$ & $28.63 \pm 2.36$ & $10.72 \pm 1.96$ & $36.33 \pm 4.14$ \\
\hline $\mathrm{NGC} 4593^{\mathrm{d}}$ & 39.5 & 1 & $9.79 \pm 0.62$ & $8.31 \pm 0.30$ & $5.56 \pm 1.27$ & $7.89 \pm 0.59$ & $8.02 \pm 1.96$ & $13.39 \pm 2.00$ \\
\hline NGC 4686 & 71.6 & $\ldots$ & $3.08 \pm 0.45$ & $2.13 \pm 0.16$ & $<0.22$ & $1.71 \pm 0.13$ & $0.53 \pm 0.09$ & $0.91 \pm 0.30$ \\
\hline NGC 526A & 81.9 & 1.5 & $5.96 \pm 0.51$ & $6.22 \pm 1.61$ & $3.94 \pm 0.47$ & $9.59 \pm 1.10$ & $3.87 \pm 0.86$ & $17.34 \pm 0.84$ \\
\hline NGC $5506^{\mathrm{d}}$ & 28.7 & 1.9 & $25.64 \pm 0.50$ & $91.75 \pm 3.31$ & $58.28 \pm 3.34$ & $152.13 \pm 9.13$ & $63.25 \pm 2.40$ & $252.82 \pm 2.29$ \\
\hline NGC 5548 & 73.5 & 1.5 & $8.08 \pm 0.50$ & $8.93 \pm 0.70$ & $1.95 \pm 0.10$ & $8.99 \pm 0.97$ & $1.73 \pm 0.75$ & $12.75 \pm 0.80$ \\
\hline
\end{tabular}


Table 1

(Continued)

\begin{tabular}{|c|c|c|c|c|c|c|c|c|}
\hline \multirow[t]{2}{*}{ Name } & \multirow[t]{2}{*}{$\begin{array}{c}\text { Distance } \\
(\mathrm{Mpc})\end{array}$} & \multirow[t]{2}{*}{ Type $^{\mathrm{a}}$} & \multirow[t]{2}{*}{$\mathrm{BAT}^{\mathrm{b}}$} & $\begin{array}{c}{[\mathrm{Ne} \text { II }]} \\
(12.81 \mu \mathrm{m})\end{array}$ & $\begin{array}{c}{[\mathrm{Ne} \mathrm{v}]} \\
(14.32 \mu \mathrm{m})\end{array}$ & $\begin{array}{c}{[\mathrm{Ne} \text { III }]} \\
(15.56 \mu \mathrm{m})\end{array}$ & $\begin{array}{c}{[\mathrm{Ne} \mathrm{v}]} \\
(24.32 \mu \mathrm{m})\end{array}$ & $\begin{array}{c}\text { [O IV] } \\
(25.89 \mu \mathrm{m})\end{array}$ \\
\hline & & & & \multicolumn{5}{|c|}{ Integrated Line Fluxes $\left(10^{-21} \mathrm{~W} \mathrm{~cm}^{-2}\right)$} \\
\hline NGC 5728d & 42.2 & 2 & $10.54 \pm 0.71$ & $30.44 \pm 1.81$ & $23.56 \pm 0.77$ & $54.76 \pm 0.51$ & $28.15 \pm 0.56$ & $118.40 \pm 7.61$ \\
\hline NGC 5995 & 108.5 & 2 & $4.51 \pm 0.61$ & $13.32 \pm 1.91$ & $7.60 \pm 1.42$ & $9.19 \pm 0.59$ & $4.61 \pm 0.84$ & $12.20 \pm 0.80$ \\
\hline NGC $6240^{g}$ & 105.4 & 2 & $7.30 \pm 0.62$ & $193.10 \pm 3.70$ & $5.10 \pm 0.90$ & $70.40 \pm 2.40$ & $<3.90$ & $27.20 \pm 0.70$ \\
\hline NGC 6860 & 63.6 & 1 & $6.50 \pm 0.73$ & $5.88 \pm 0.11$ & $2.95 \pm 0.94$ & $7.50 \pm 0.53$ & $2.84 \pm 0.21$ & $11.70 \pm 0.39$ \\
\hline NGC $7172^{d}$ & 33.9 & 2 & $18.11 \pm 0.70$ & $32.03 \pm 2.44$ & $8.99 \pm 0.80$ & $15.71 \pm 0.69$ & $11.26 \pm 0.95$ & $42.60 \pm 3.44$ \\
\hline NGC $7213^{d}$ & 22.0 & 1.5 & $5.75 \pm 0.67$ & $27.47 \pm 1.33$ & $0.56 \pm 0.01$ & $12.77 \pm 0.66$ & $<1.94$ & $2.75 \pm 0.59$ \\
\hline NGC $7314^{d}$ & 18.3 & 1.9 & $4.63 \pm 0.59$ & $8.97 \pm 0.74$ & $16.84 \pm 0.60$ & $23.28 \pm 0.41$ & $21.92 \pm 0.40$ & $69.62 \pm 7.82$ \\
\hline NGC $7469^{c}$ & 69.8 & 1.2 & $6.66 \pm 0.44$ & 200.00 & 11.60 & 34.00 & 14.70 & 43.00 \\
\hline NGC $7582^{d}$ & 22.0 & 2 & $7.92 \pm 0.55$ & $250.94 \pm 3.53$ & $38.02 \pm 3.86$ & $104.99 \pm 4.37$ & $59.54 \pm 6.39$ & $227.65 \pm 10.88$ \\
\hline NGC 7603 & 127.6 & 1.5 & $4.70 \pm 0.51$ & $9.32 \pm 0.72$ & $0.47 \pm 0.01$ & $5.07 \pm 0.49$ & $<0.38$ & $3.34 \pm 0.18$ \\
\hline NGC 7682 & 73.4 & 2 & 2.27 & $5.46 \pm 0.25$ & $1.98 \pm 0.19$ & $8.07 \pm 0.15$ & $3.69 \pm 1.46$ & $16.21 \pm 0.66$ \\
\hline UGC 03601 & 73.3 & 1.5 & $4.38 \pm 0.67$ & $5.26 \pm 0.53$ & $2.01 \pm 0.12$ & $7.60 \pm 0.13$ & $1.79 \pm 0.44$ & $13.45 \pm 0.65$ \\
\hline UGC 06728 & 27.7 & 1.2 & $2.95 \pm 0.37$ & $1.40 \pm 0.36$ & $1.16 \pm 0.10$ & $1.38 \pm 0.05$ & $3.24 \pm 0.03$ & $4.61 \pm 0.81$ \\
\hline UGC 12282 & 72.7 & $\ldots$ & $2.49 \pm 0.50$ & $2.08 \pm 0.28$ & $0.50 \pm 0.01$ & $1.76 \pm 0.05$ & $1.44 \pm 0.20$ & $4.07 \pm 0.37$ \\
\hline UGC 12741 & 74.7 & $\ldots$ & $4.00 \pm 0.59$ & $1.72 \pm 0.07$ & $0.39 \pm 0.06$ & $1.55 \pm 0.05$ & $0.65 \pm 0.06$ & $3.58 \pm 0.38$ \\
\hline
\end{tabular}

Notes.

a AGN types are taken from NED.

b The BAT flux is presented in units of $10^{-11} \mathrm{erg} \mathrm{s}^{-1} \mathrm{~cm}^{-2}$.

c Weedman et al. (2005).

${ }^{\mathrm{d}}$ Distances are taken from EDD.

e From the 9 month BAT survey (Tueller et al. 2008).

f Tommasin et al. (2008).

g Armus et al. (2006).

linearity correction, and wavelength and flux calibrations. ${ }^{14}$ Many of the sources in our sample have dedicated off-source observations to do sky subtraction, which can alleviate the effect of rogue pixels and variable background. Off-source images were averaged for each node position and order to obtain a final background image. Then, we subtract the sky background from our spectrum. The full slit spectra were extracted from the IRS data using the Spectroscopy Modeling Analysis and Reduction Tool (SMART) v6.4.0 (Higdon et al. 2004). For the extraction we used the "Full Aperture" extraction method for high-resolution observations of point sources. We created median basic calibrated data files from each node and then the spectra from each node position for $\mathrm{SH} / \mathrm{LH}$ were averaged using $2.5 \sigma$ clipping to reject outliers. Finally, we trimmed the edges of the orders to obtain a clean spectrum. For sources without dedicated off-source observations we did not perform any background subtraction because we only required emissionline fluxes; furthermore, our hard X-ray selected sample is characterized by bright nuclear sources which fill the highresolution slit resulting in a minimal background correction. We performed the line fit with SMART using a polynomial to fit the continuum and a Gaussian for the line profile. In Table 1 , we report the line fluxes, together with their $1 \sigma$ statistical error for the whole high-resolution sample. The typical $1 \sigma$ statistical errors for the mission lines presented in this work are, on average, $\sim 9 \%, \sim 10 \%, \sim 5 \%, \sim 12 \%$, and $\sim 8 \%$ for the [Ne II] $12.81 \mu \mathrm{m},[\mathrm{Ne} \mathrm{v}] 14.32 \mu \mathrm{m}$, [Ne III] $15.56 \mu \mathrm{m}$, $[\mathrm{Ne} \mathrm{v}] 24.32 \mu \mathrm{m}$, and [O Iv] emission lines, respectively. From this and for the sake of simplicity we did not plot error bars on individual objects in the different comparisons presented in this work, as the errors are comparable to the symbol size uses in the figures. For non-detections we quote the $3 \sigma$ upper limits

\footnotetext{
${ }^{14}$ See the IRS Pipeline Handbook, http://ssc.spitzer.caltech.edu/irs/.
}

as defined for emission lines with an $\mathrm{S} / \mathrm{N}$ less than 3. Finally, the emission line fluxes are presented here without reddening corrections.

Each of the galaxies in our sample has clearly detected [O IV], [Ne II], and [Ne III] emission. However, [Ne v] $14.32 \mu \mathrm{m}$ and $24.32 \mu \mathrm{m}$ were not detected in $\sim 10 \%$ and $\sim 15 \%$ of the sources, respectively. Similar results are obtained for the [Ne v] $14.32 \mu \mathrm{m}$ and $24.32 \mu \mathrm{m}$ in the Spitzer high-resolution spectroscopy of the $12 \mu \mathrm{m}$ Seyfert galaxies (Tommasin et al. $2008,2010)$. The importance of [O IV] and the neon emission lines is that they are sufficient to distinguish between stellar and AGN activity. For example, the [Ne III]/[Ne II] and [O IV]/ [Ne II] ratios are good discriminators of star formation and AGN emission (Genzel et al. 1998; Sturm et al. 2002; Satyapal et al. 2004; Meléndez et al. 2008a), while the strengths of [O IV] (Meléndez et al. 2008b; Rigby et al. 2009) and [Ne v] (e.g., Satyapal et al. 2007; Dudik et al. 2007; Abel \& Satyapal 2008) scale with the luminosity of the AGNs. Moreover, the neon line ratios are also tracers that are insensitive to abundance. It should be noted, however, that [O IV] and [Ne v] can be weak in some classical AGNs (Weedman et al. 2005), notably ultraluminous infrared galaxies (ULIRGs), possibly due to the shielding of the narrow line region by optically thick, dusty gas close to the AGNs (e.g., Armus et al. 2007).

\section{MID-INFRARED PROPERTIES OF THE BAT SAMPLE}

\subsection{Comparison of the IR Emission Line and BAT Luminosities}

As noted above, via the hard X-ray band we can detect AGNs that may have been missed in optical surveys due to the extinction of their optical emission lines. Among the 79 targets presented here (BAT AGNs), we have found six poorly studied or previously unknown AGNs ("new BAT-detected AGN"): ESO 005-G004, MRK 18, NGC 973, NGC 4686, UGC 12282, and 

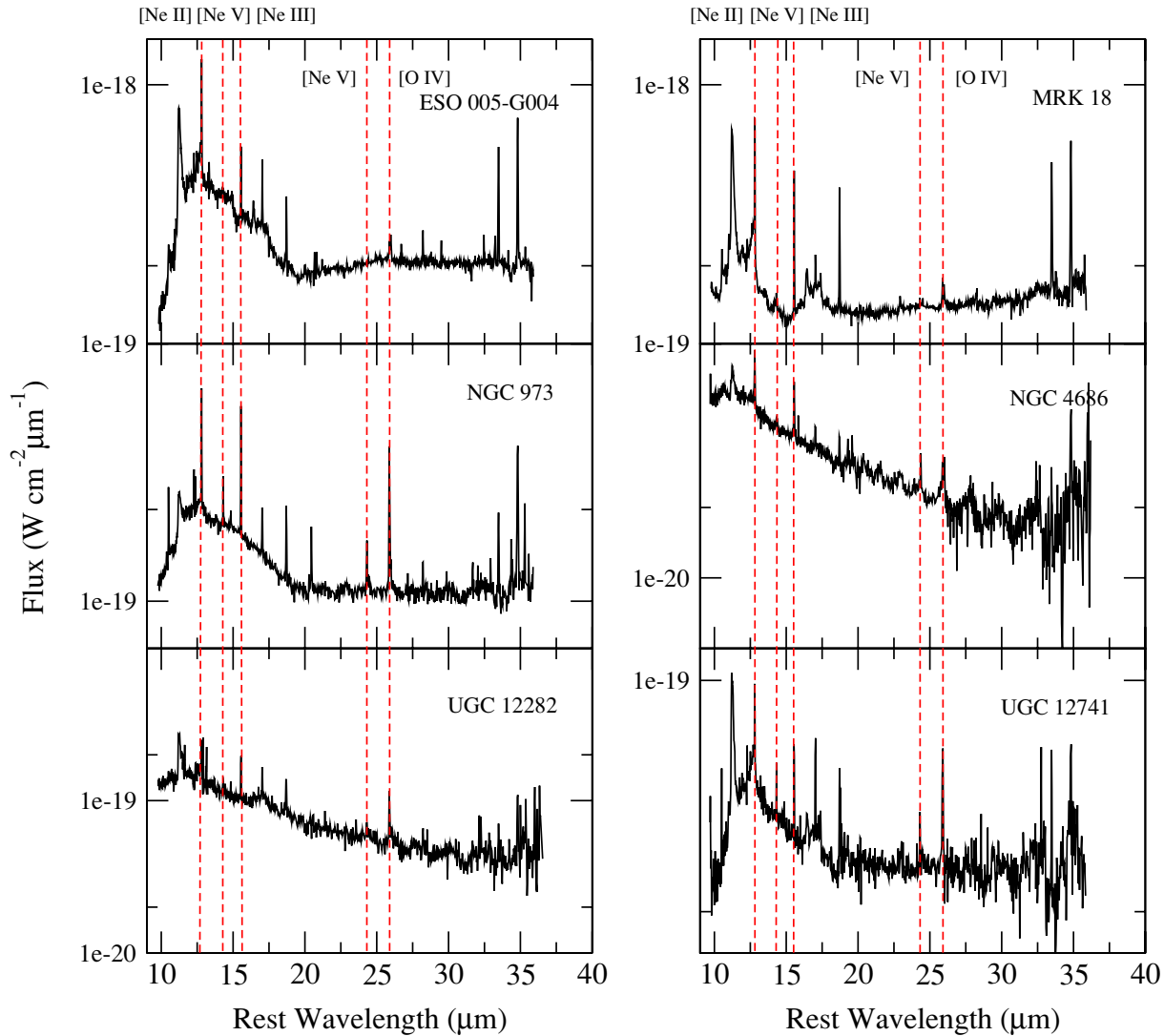

Figure 1. Combined Spitzer IRS SH and LH spectra for the newly detected AGNs in the BAT sample. The [Ne II] $12.81 \mu \mathrm{m},[\mathrm{Ne} I I I] 15.56 \mu \mathrm{m},[\mathrm{Ne} \mathrm{v}] 14.32 \mu \mathrm{m}$, [Ne v] $24.32 \mu \mathrm{m}$, and [O IV] $25.89 \mu \mathrm{m}$ lines are indicated by the vertical lines. The strong emission line features at $\sim 33 \mu \mathrm{m}$ and $\sim 35 \mu \mathrm{m}$ correspond to [S III] and [Si II], respectively.

(A color version of this figure is available in the online journal.)
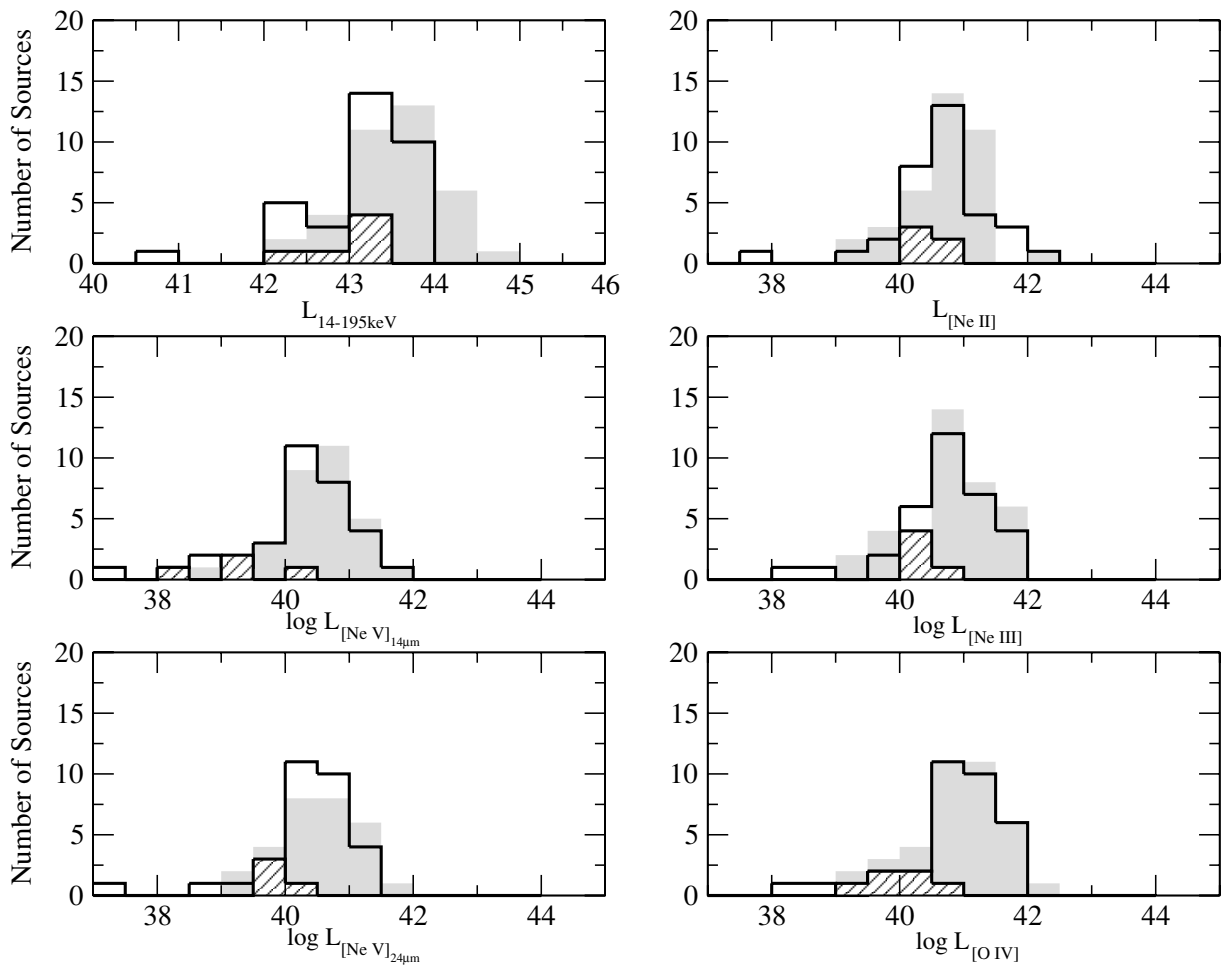

Figure 2. Histograms for the mid-infrared and BAT (14-195 keV) luminosities for our sample. Seyfert 1 and Seyfert 2 galaxies are indicated with gray bins and solid lines, respectively. The newly detected BAT AGNs are indicated in bins with dashed lines; note that their [Ne III] and [O IV] luminosities appear to be low, i.e., compared to AGNs with similar BAT luminosities. The K-S test for these emission line luminosities shows that two samples drawn from the same population would differ this much $\sim 6.3 \%, \sim 82.6 \%, \sim 69.8 \%, \sim 50.9 \%, \sim 28.6 \%$, and $\sim 98.1 \%$ of the time for the BAT, [Ne II], [Ne v] $14 \mu \mathrm{m}$, [Ne III], [Ne v] $24 \mu \mathrm{m}$, and [O IV] luminosity distributions, respectively. 

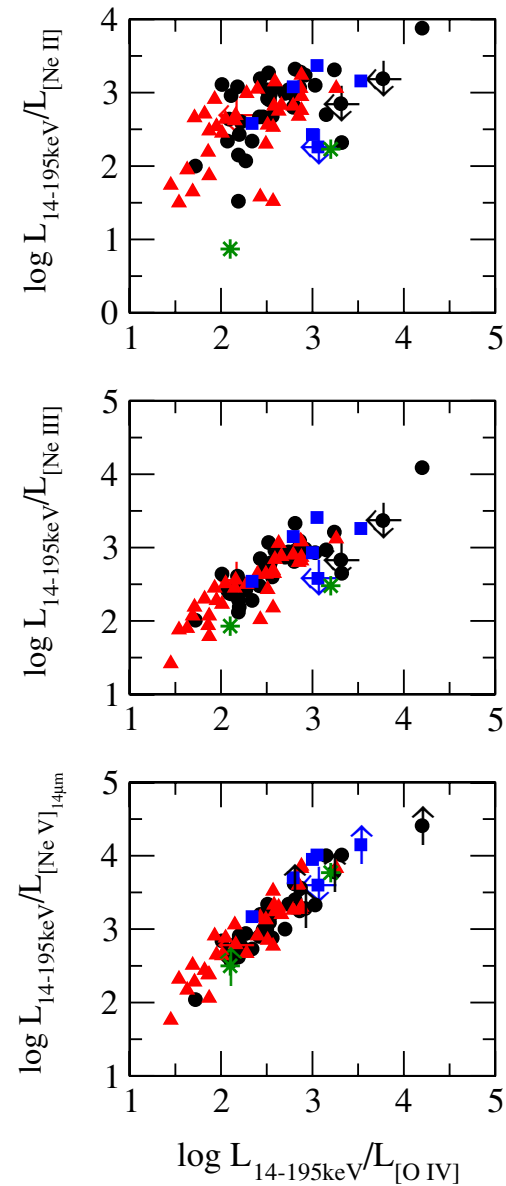

Figure 3. Comparison between the ratios of the BAT band and the mid-infrared emission lines. Seyfert 1 galaxies are presented as black circles, Seyfert 2 galaxies are red triangles, blue squares represent the newly detected BAT AGNs and green stars are LINERs. Of particular interest is the statistically significant (see Table 3) separation between Seyfert types in the $L_{14-195 \mathrm{keV}} / L_{[\mathrm{O} \text { Iv] }}$ ratio, in agreement with this ratio being an indicator of Compton scattering in the BAT band. One must note that below $L_{\mathrm{BAT}} /[\mathrm{O}$ IV] $<\sim 2.0$ there are only two Seyfert 1 galaxies but 13 Seyfert 2 galaxies ( $\sim 40 \%$ of the Seyfert 2 population). (A color version of this figure is available in the online journal.)

UGC 12741. There have been attempts to classify some of these galaxies. Of these, ESO 005-G004 has been classified as a Seyfert 2, based on its X-ray properties although no optical AGN signature has been detected (Landi et al. 2007; Ueda et al. 2007). Based on follow-up observations in the optical, MRK 18 has an ambiguous classification with $\mathrm{H}$ II/LINER properties (Winter et al. 2010) and NGC 973 has been classified as a narrow emission line AGN with Sy2/LINER properties (Masetti et al. 2008). UGC 12282 has been classified as an Sy 1.9 based on its optical spectra (Véron-Cetty \& Véron 2006). Finally, to the best of our knowledge, there is no assigned classification for NGC 4686 and UGC 12741. The Spitzer IRS spectra of these objects are shown in Figure 1.

In Figures 2-7, we compare the mid-infrared emission lines and the BAT luminosities (and fluxes), correlations between the emission lines, and emission line ratios. The statistical analysis for these plots is listed in Table 2, which includes the Spearman rank order correlation coefficient with its associated null probability, the generalized Kendall's correlation coefficient for censored data and the Kendall's coefficient for partial correlation with censored data. One should note that, due to redshift effects, luminosity-luminosity plots will almost always show some correlation. Thus, we are primarily interested in the dispersion of the correlations or the slopes. Furthermore, caution must be taken when applying statistical analysis to data sets that contain non-detections (upper limits), or "censored" data points. To deal with these problems we have used Astronomy SURVival analysis (ASURV) Rev 1.2 (Isobe \& Feigelson 1990), which implement the methods presented in Isobe et al. (1986). We also used a test for partial correlation with censored data (Akritas \& Siebert 1996) in order to exclude the redshift effect in the correlations.

In Figure 2, we show the distribution of the observed [Ne II], [Ne III], [Nev], [O IV], and BAT luminosities $\left(L_{\mathrm{BAT}}\right)$ for our sample. The results of the K-S tests, comparing Seyfert 1s and $2 \mathrm{~s}$, for these quantities are listed in Table 3. This table also includes information about the numbers of Seyfert 1 and Seyfert 2 galaxies, median values, and standard deviations of the mean for the measured quantities. As is apparent from both the histograms and the K-S results, the two Seyfert types are statistically indistinguishable, i.e., there are essentially no differences between Seyfert 1s and 2s when we directly compare the BAT luminosities or any of the IR line strengths. These results are in agreement with the similar optical luminosities found for broad and narrow line sources, with optical Seyfert classifications, in the most recent study on the optical spectral properties of the BAT AGNs (Winter et al. 2010). The smallest null-test probability is for $L_{\mathrm{BAT}}$, which, as we will discuss below, is evidence that Seyfert $2 \mathrm{~s}$ suffer more from Compton scattering in the BAT band. Although there were too few of the "new BAT AGN" to separate them for a K-S on these quantities, the histograms indicate that they are relatively weaker in their observed mid-infrared emission line luminosities, compared to their BAT luminosities.

As mentioned before, a detailed statistical analysis for the different correlations between the BAT and mid-infrared luminosities is presented in Table 2. From this analysis the weaker correlation found in the [Ne II]-BAT relationship could be the result of active star formation contributing to the [Ne II] emission line in some of the AGNs (e.g., Sturm et al. 2002; Schweitzer et al. 2006; Ho \& Keto 2007; Meléndez et al. 2008a). On the other hand, stronger correlations are found between [NeIII], [Ne v], and [O IV] luminosities when compared to $L_{\mathrm{BAT}}$, suggesting that, on average, there is no strong enhancement due to star formation in the [Ne III] and [O IV] emission in the BAT sample.

In Table 4, we present the linear regression fits for all correlations. The somewhat steeper slopes for the Seyfert $2 \mathrm{~s}$ are consistent with the decrement in the observed X-ray emission in the BAT band due to the effect of Compton scatter. The strong similarities between Seyfert types lessen when we compare the ratios of mid-infrared line strengths and the $\mathrm{X}$-ray continuum strength. In Figure 3, we show the ratios of $L_{\mathrm{BAT}}$ and [Ne II], [Ne III], and [Ne v] $14.32 \mu \mathrm{m}$ compared to $L_{\mathrm{BAT}} /\left[\mathrm{O}\right.$ IV]. The histograms for the ratios of $L_{\mathrm{BAT}}$ and [Ne II], [Ne III], [Ne v] 14.32/24.32 $\mu \mathrm{m}$ and [O IV] are presented in Figure 4. Of particular interest in these comparisons is that below $\log L_{\mathrm{BAT}} /[\mathrm{O}$ IV $]<\sim 2.0$ there are only two Seyfert 1 galaxies but 13 Seyfert 2 galaxies ( $\sim 40 \%$ of the Seyfert 2 population in our sample). Therefore, in these plots, one can see that the Seyfert $2 \mathrm{~s}$ have lower ratios of $L_{\mathrm{BAT}}$ to the emission lines compared to the Seyfert $1 \mathrm{~s}$, which is, we believe is due to the effect of Compton scattering in the BAT band in some Seyfert 2 galaxies. This result is in agreement with the K-S test null probability for this ratio (see Table 3). 

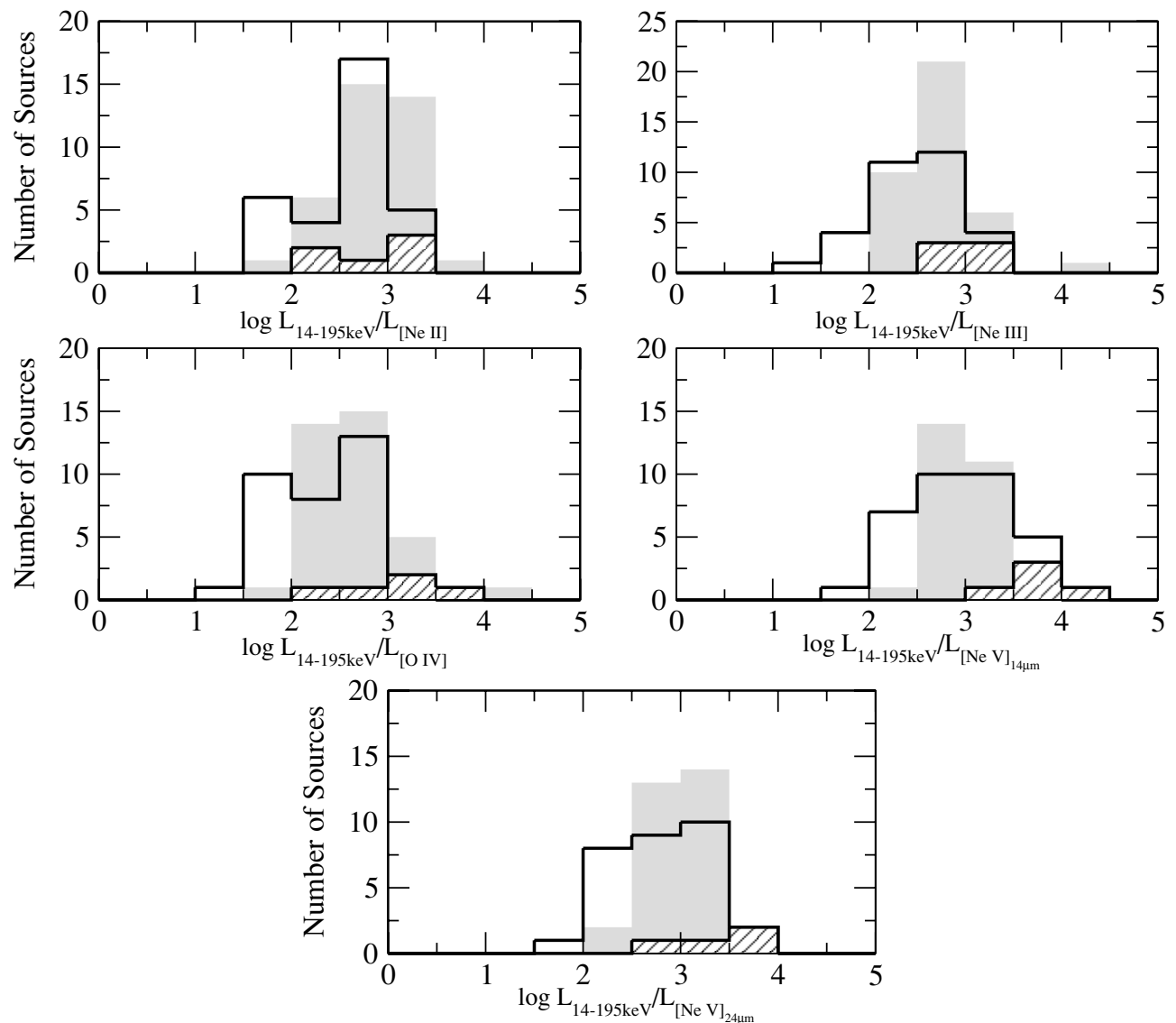

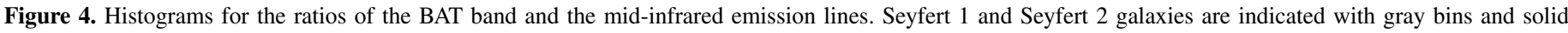

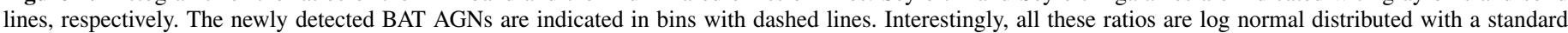

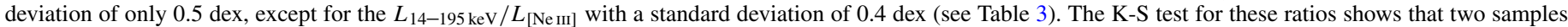

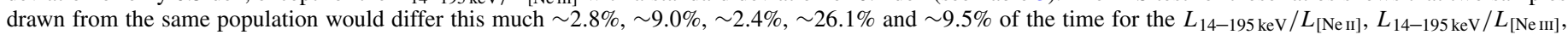

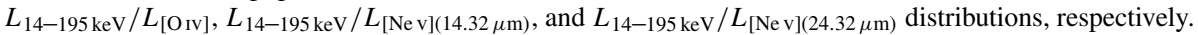

Similarly, there is only one Seyfert 2 galaxy, NGC 4138, with $\log L_{\mathrm{BAT}} /[\mathrm{O}$ IV $] \gtrsim 3.0$.

From the $L_{\mathrm{BAT}} /[\mathrm{O}$ IV] ratios, the lowest values, likely due to the effects of Compton scattering in the BAT band are NGC 1365, NGC 2992, NGC 3281, NGC 7582, MRK 3, and MCG-03-34-064. Note that NGC 2992 and NGC 1365 are very variable in the $\mathrm{X}$-ray, which can contribute to their low $L_{\mathrm{BAT}} /\left[\mathrm{O}\right.$ IV] and $L_{\mathrm{BAT}} /[\mathrm{Ne} \mathrm{v}]$ ratios (see Gilli et al. 2000; Risaliti et al. 2000b). In the BAT spectra (R. F. Mushotzky et al. 2010, in preparation), MRK 3 shows a flat spectrum with a photoelectric cutoff, consistent with a high column density, but not a Compton thick absorber. On the other hand, NGC 1365, NGC 3281, and NGC 7582 are most probably Compton thick sources and the BAT data for NGC 2992 and MCG-03-34064 has a low $\mathrm{S} / \mathrm{N}$ which makes it difficult to constrain the spectrum well. These results suggest that the low BAT to [O IV] ratio is a marker for very high column densities toward the $\mathrm{X}$-ray source, in agreement with previous studies (Meléndez et al. 2008b; Rigby et al. 2009; La Massa et al. 2009). These results argue in favor of the unified model of active galaxies, where the amount of X-ray emission suppressed, relative to an isotropic indicator for the AGN power, is related to the absorbing column density toward the X-ray source (see also Bassani et al. 1999; Heckman et al. 2005; Netzer et al. 2006). Given the high ionization potential for $\mathrm{Ne}^{+4}(\sim 97 \mathrm{eV})$, the mid-infrared [Ne v] emission lines are claimed to be an unambiguous signatures of an AGN (e.g., Satyapal et al. 2007, 2009). Therefore, the very tight correlation between the $L_{\mathrm{BAT}} /[\mathrm{O} \mathrm{IV}]$ and $L_{\mathrm{BAT}} /[\mathrm{Ne} \mathrm{v}]$ $14.32 \mu \mathrm{m}$ ratio confirm that [O IV] is AGN-driven in this sample.

In Figure 5, we plot both the flux and luminosity of [Ne II], [Ne III] and [O IV] against those of [Ne v] $14.32 \mu \mathrm{m}$. The very tight correlation between $[\mathrm{Ne} \mathrm{v}]$ and [O IV] suggests that both of these lines are produced by the same physical process, i.e., photoionization by the AGN continuum (see also, Meléndez et al. 2008a, 2008b). This is also true for the tight correlation between [Ne v] and [Ne III] found in our sample. While there is some scatter for [Ne III], which may also be due to star formation (Ho \& Keto 2007), the tightness of the correlation suggests that [Ne III] is primarily produced by the AGN in these objects (see also Gorjian et al. 2007; Goulding \& Alexander 2009), in agreement with the good correlation found between the [Ne III] and BAT luminosity. Furthermore, the tightness of these correlations, especially in flux-flux, suggests that the constant mid-infrared ratios observed are not dominated by aperture effects. The extraction aperture for the $[\mathrm{O}$ IV $]$ is bigger than that for the [Ne III] and [Ne v] $14 \mu \mathrm{m}$, implying that the emitting regions where these lines originate are well within the central kpc of these sources, in agreement with our previous photoionization studies (e.g., Meléndez et al. 2008a, 2008b). We found that none of the correlations presented, e.g., between the mid-infrared lines and the BAT luminosities, are driven by distances effects, as shown by the partial correlation analysis (see Table 2) and in agreement with the tightness of the flux-flux correlations. Finally, the largest scatter occurs for [Ne II], which 

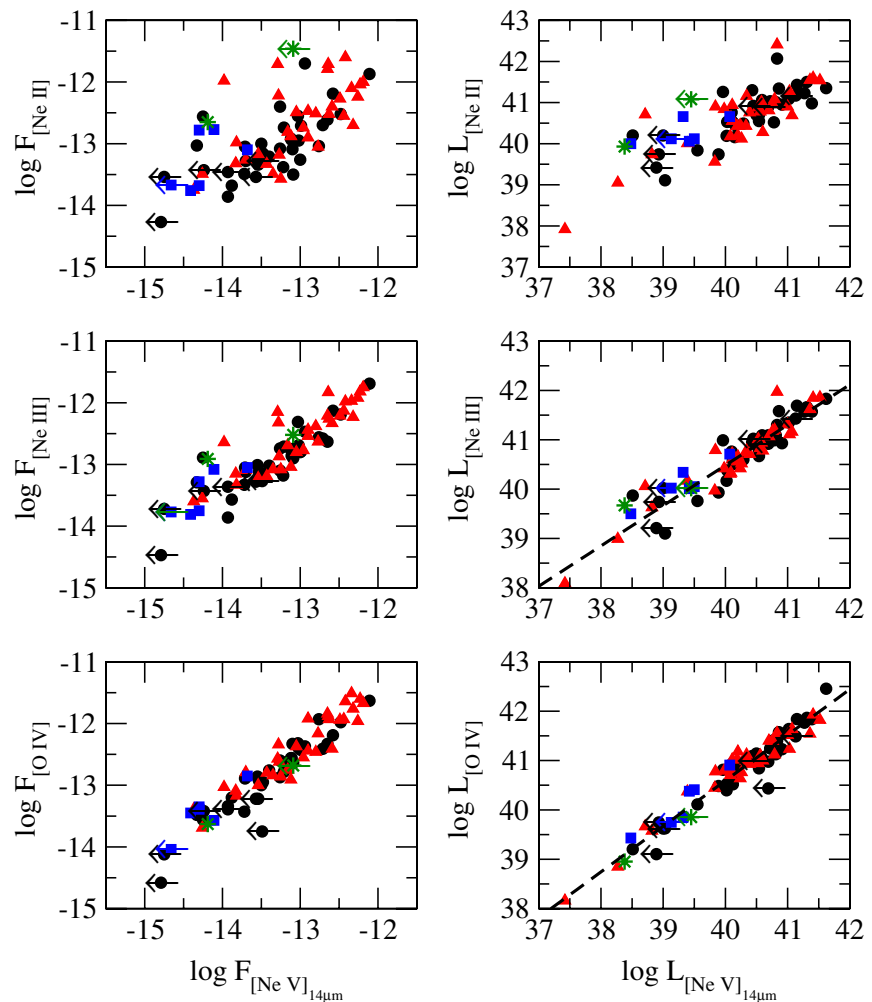

Figure 5. Correlation between [Ne II],[Ne III], [Ne v]14 $\mu \mathrm{m}$, and [O IV] fluxes and luminosities. Symbols are the same as in Figure 3. The dashed line represents the linear regression for the full sample (see Table 4). The statistical analysis and linear regression fits for these correlation are presented in Tables 2 and 4, respectively.

(A color version of this figure is available in the online journal.)

is consistent with the interpretation that this line may contain a significant contribution from stellar processes, resulting in the enhancement of this emission line, as also evident in Figure 3. As we discussed before, the $[\mathrm{Ne} \mathrm{v}]$ emission lines are good indicators for the AGN power, therefore, the good correlation found between [ $\mathrm{Ne}$ II] and [Ne V] (see Table 2) suggest that, on average, the observed [Ne II] emission in the BAT sample is still dominated by the AGNs.

As we mentioned before, the ratios of high-ionization lines to low-ionization lines can reveal the relative contributions of the AGNs and star formation. In Figure 6, we show the ratios of $[\mathrm{Ne} \mathrm{III}] /[\mathrm{Ne} \mathrm{II}]$ and $[\mathrm{NeV}] 14 \mu \mathrm{m} /[\mathrm{Ne} \mathrm{II}]$ compared to $[\mathrm{O}$ IV] $/[\mathrm{Ne}$ II] for the BAT sample. One result, which was also discussed in Meléndez et al. (2008a), is that $\sim 62 \%$ and $\sim 72 \%$ of our AGNs have [Ne III]/[Ne II] and [O IV]/[Ne II] ratios, respectively, greater than unity. This suggests, again, that in the BAT targets, the mid-infrared emission lines are dominated by the AGNs. These results are also in agreement with the high-resolution Spitzer analysis of the $12 \mu \mathrm{m}$ sample by Tommasin et al. $(2008,2010)$ where they found the same range for the $[\mathrm{Ne} \mathrm{III}] /[\mathrm{Ne} \mathrm{II}]$ and $[\mathrm{O} \mathrm{IV}] /[\mathrm{Ne} \mathrm{II}]$ ratios. However, the $\mathrm{K}-\mathrm{S}$ test for the $[\mathrm{Ne} \mathrm{III}] /[\mathrm{Ne} \mathrm{II}]$ ratio between the $12 \mu \mathrm{m}$ sample and the BAT sample returns a $\sim 1.9 \%$ probability of the null hypothesis, meaning that these samples are statistically different in their stellar content, with galaxies in the $12 \mu \mathrm{m}$ sample having slightly smaller ratios on average, $1.1 \pm 0.9$, than that found for the BAT sample, $1.3 \pm 0.8$. On the other hand, the K-S test for the $\left[\mathrm{O}_{\mathrm{IV}}\right] /[\mathrm{Ne} \mathrm{III}]$ ratio between the $12 \mu \mathrm{m}$ sample and the BAT sample returns a $\sim 26.5 \%$ probability of the null hypothesis. These results suggest that even the $12 \mu \mathrm{m}$ sample could be
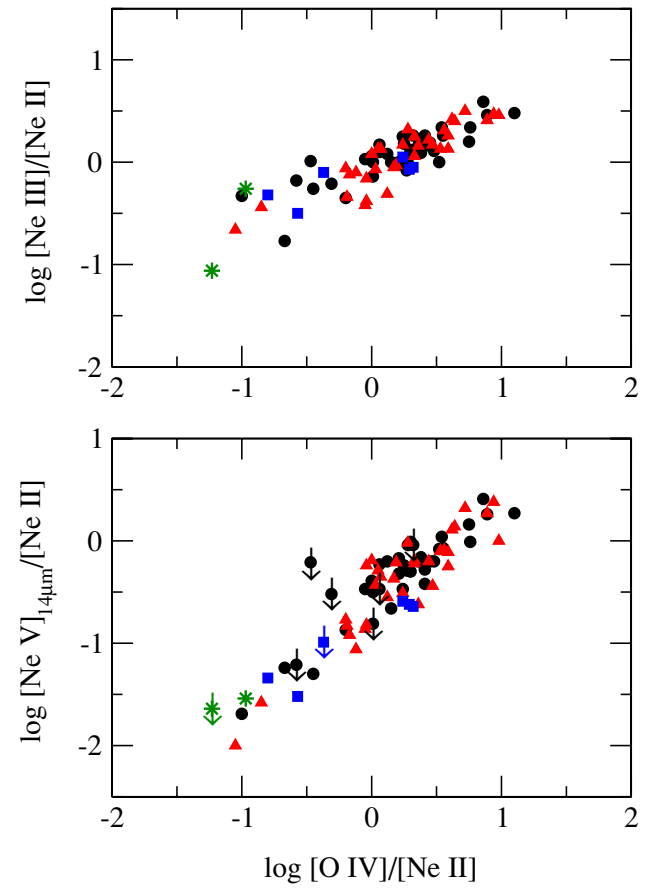

Figure 6. $[\mathrm{Ne}$ III] $/[\mathrm{Ne}$ II] and $[\mathrm{Ne} \mathrm{v}] 14 \mu \mathrm{m} /[\mathrm{Ne}$ II] vs. [O IV] $/[\mathrm{Ne}$ II $]$ ratios for Seyfert 1, Seyfert 2, BAT-detected AGNs, and LINERs in the BAT sample. Symbols are the same as in Figure 3. The $\sim 62 \%$ and $\sim 72 \%$ of the objects in our sample have $[\mathrm{Ne} \mathrm{III}] /[\mathrm{Ne} \mathrm{II}]$ and $[\mathrm{O} \mathrm{IV}] /[\mathrm{Ne} \mathrm{II}]$ have ratios bigger than unity, which has been shown to be typical of AGN-ionized emission-line gas (e.g., Meléndez et al. 2008a). The [Ne v] and [O IV] ratios appear well correlated, as expected given the tight correlation seen in Figure 5.

(A color version of this figure is available in the online journal.)

subject to recent star formation contamination, as mapped through the [Ne II] enhancement, in agreement with previous considerations (Hunt \& Malkan 1999). This mild contamination due to star formation becomes noticeable when comparing the $12 \mu \mathrm{m}$ sample with the $14-195 \mathrm{keV}$ sample, the latter of which is less biased toward star-forming systems. Nonetheless, the similarity on their $[\mathrm{OIV}] /[\mathrm{Ne} \mathrm{III}]$ ratios suggests that both samples have a strong AGN contribution to their observed narrow line emission.

The tight correlation between [O IV] and [ $\mathrm{Ne} \mathrm{v}]$, evident in Figure 5 , is seen here as well. The $[\mathrm{Ne} \mathrm{V}] /[\mathrm{Ne}$ II] versus [O IV]/ [Ne II] plot shows a number of sources, including several of the newly BAT-detected AGN and the two LINERs in the sample, with relatively weak high-ionization lines, which can result from a weak AGN (e.g., Netzer et al. 2004), strong nuclear star formation (e.g., Meléndez et al. 2008a), extinction toward the NLR region (e.g., Netzer et al. 2006), or shielding of the NLR from the ionizing continuum (e.g., Armus et al. 2007). Comparing the $[\mathrm{Ne}$ III]/[Ne II] to the [Ne V] $14.32 \mu \mathrm{m} /[\mathrm{Ne}$ II] we found the same overall trend, however, the slope of the former flattens toward lower ratios of [O IV]/[Ne II] possible due to the contribution of star formation to the [Ne III]. Interestingly, all these ratios are log normal distributed with a standard deviation of only $\sim 0.3$ dex, except for the $[\mathrm{NeV}] 14.32 \mu \mathrm{m} /[\mathrm{O}$ Iv] with a standard deviation of $\sim 0.2$ dex. The $[\mathrm{O}$ IV] $/[\mathrm{Ne}$ II] emission line ratio was also compared with the redshift $(z)$ in order to check if our previous results are being affected by aperture affects, in other words, if our results are biased toward small [Ne III]/ $[\mathrm{Ne}$ II $]$ and $[\mathrm{O}$ IV $] /[\mathrm{Ne}$ II] ratios at higher redshifts. We found that the Spearman rank, $\rho_{s}=0.129 ; P_{\rho}=0.30$, and Kendall test, $\tau=0.08 ; P_{\tau}=0.27$, did not show any correlation with $z$. A similar result was obtained for the $\left[\mathrm{O}_{\mathrm{IV}}\right] /[\mathrm{Ne}$ III $]$ ratios and 

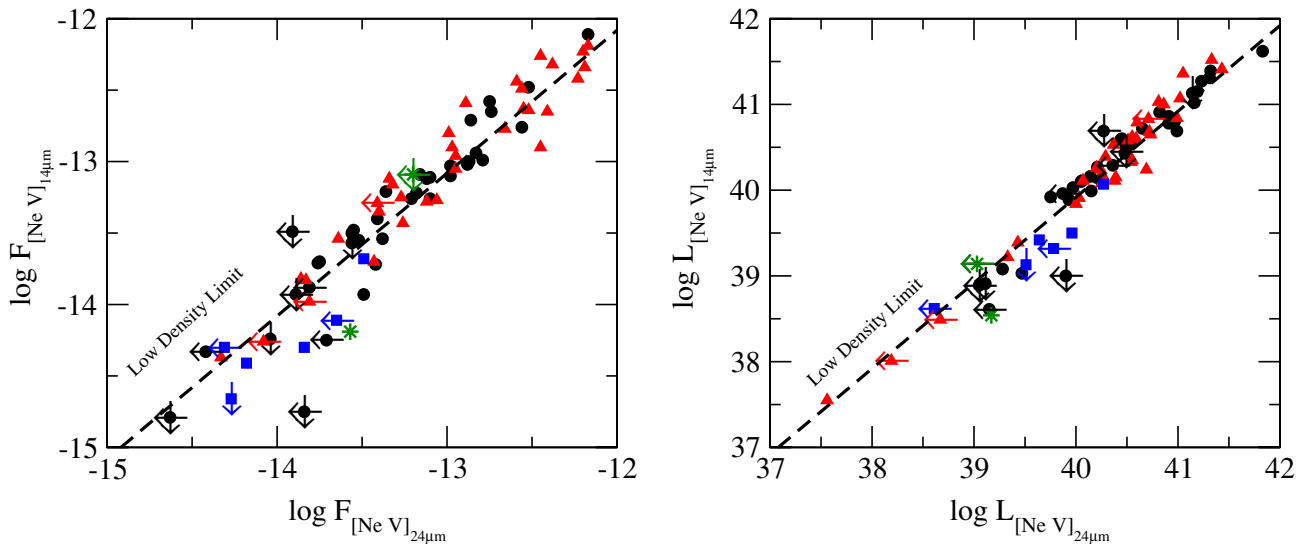

Figure 7. Correlation between the [Ne v] $24.32 \mu \mathrm{m}$ and [Ne v] $14.32 \mu \mathrm{m}$ fluxes and luminosities for our X-ray selected sample. Symbols are the same as in Figure 3 . The low-density limit for this ratio is given by the dashed line; AGNs lying below and to the right have [Ne v] $14.32 \mu \mathrm{m} /[\mathrm{Ne} \mathrm{v}] 24.32 \mu \mathrm{m}$ ratios smaller than the theoretical limit. The majority of the newly detected BAT AGNs have ratios below the limit, implying dust extinction toward the [Ne v]14 $\mu \mathrm{m}$ emitting region.

(A color version of this figure is available in the online journal.)

Table 2

Statistical Analysis for the Different Relationships Between the Mid-infrared Emission Lines and BAT Luminosity for the Sample

\begin{tabular}{|c|c|c|c|c|c|c|c|}
\hline Variables & $\rho_{s}$ & $P_{\rho}$ & $\tau$ & $P_{\tau}$ & $\tau_{p}$ & $\sigma$ & $P_{\tau}(p)$ \\
\hline \multicolumn{8}{|l|}{ All Sample } \\
\hline BAT-[Ne II] & 0.66 & $<1 \times 10^{-6}$ & 0.49 & $<1 \times 10^{-6}$ & 0.37 & 0.07 & $<1 \times 10^{-6}$ \\
\hline BAT-[Ne III] & 0.76 & $<1 \times 10^{-6}$ & 0.58 & $<1 \times 10^{-6}$ & 0.45 & 0.06 & $<1 \times 10^{-6}$ \\
\hline BAT-[O IV $]$ & 0.65 & $<1 \times 10^{-6}$ & 0.48 & $<1 \times 10^{-6}$ & 0.36 & 0.06 & $<1 \times 10^{-6}$ \\
\hline BAT- $[\mathrm{Ne} \mathrm{v}]_{14 \mu \mathrm{m}}$ & 0.63 & $<1 \times 10^{-6}$ & 0.50 & $<1 \times 10^{-6}$ & 0.38 & 0.07 & $<1 \times 10^{-6}$ \\
\hline$[\mathrm{Ne}$ III]-[Ne II] & 0.89 & $<1 \times 10^{-6}$ & 0.74 & $<1 \times 10^{-6}$ & 0.68 & 0.06 & $<1 \times 10^{-6}$ \\
\hline [O IV $]-[\mathrm{Ne}$ II $]$ & 0.80 & $<1 \times 10^{-6}$ & 0.60 & $<1 \times 10^{-6}$ & 0.54 & 0.06 & $<1 \times 10^{-6}$ \\
\hline [O IV]-[Ne III] & 0.94 & $<1 \times 10^{-6}$ & 0.80 & $<1 \times 10^{-6}$ & 0.76 & 0.06 & $<1 \times 10^{-6}$ \\
\hline$[\mathrm{Ne} \mathrm{v}]_{14 \mu \mathrm{m}}-[\mathrm{Ne} \mathrm{II}]$ & 0.77 & $<1 \times 10^{-6}$ & 0.59 & $<1 \times 10^{-6}$ & 0.53 & 0.06 & $<1 \times 10^{-6}$ \\
\hline$[\mathrm{Ne} \mathrm{v}]_{14 \mu \mathrm{m}}-[\mathrm{Ne} \mathrm{III}]$ & 0.91 & $<1 \times 10^{-6}$ & 0.79 & $<1 \times 10^{-6}$ & 0.75 & 0.06 & $<1 \times 10^{-6}$ \\
\hline$[\mathrm{Ne} \mathrm{v}]_{14 \mu \mathrm{m}}-[\mathrm{O} \mathrm{IV}]$ & 0.94 & $<1 \times 10^{-6}$ & 0.80 & $<1 \times 10^{-6}$ & 0.77 & 0.06 & $<1 \times 10^{-6}$ \\
\hline$[\mathrm{Ne} \mathrm{v}]_{24 \mu \mathrm{m}}-[\mathrm{Ne} \mathrm{v}]_{14}$ & 0.93 & $<1 \times 10^{-6}$ & 0.80 & $<1 \times 10^{-6}$ & 0.77 & 0.06 & $<1 \times 10^{-6}$ \\
\hline \multicolumn{8}{|l|}{ Seyfert 1 Galaxies } \\
\hline BAT-[Ne II] & 0.75 & $<1 \times 10^{-6}$ & 0.60 & $<1 \times 10^{-6}$ & 0.37 & 0.11 & $<9 \times 10^{-4}$ \\
\hline BAT-[Ne III] & 0.82 & $<1 \times 10^{-6}$ & 0.66 & $<1 \times 10^{-6}$ & 0.41 & 0.12 & $<1 \times 10^{-3}$ \\
\hline BAT-[O IV $]$ & 0.72 & $<1 \times 10^{-6}$ & 0.56 & $<1 \times 10^{-6}$ & 0.33 & 0.11 & $<3 \times 10^{-3}$ \\
\hline BAT-[Ne v] & 0.61 & $<2 \times 10^{-4}$ & 0.52 & $<1 \times 10^{-6}$ & 0.32 & 0.12 & $<8 \times 10^{-3}$ \\
\hline$[\mathrm{O}$ IV $]-[\mathrm{Ne}$ II $]$ & 0.84 & $<1 \times 10^{-6}$ & 0.66 & $<1 \times 10^{-6}$ & 0.54 & 0.09 & $<1 \times 10^{-6}$ \\
\hline [O IV $]-[\mathrm{Ne}$ III] & 0.93 & $<1 \times 10^{-6}$ & 0.79 & $<1 \times 10^{-6}$ & 0.71 & 0.08 & $<1 \times 10^{-6}$ \\
\hline$[\mathrm{Ne} \mathrm{V}]_{14 \mu \mathrm{m}}-[\mathrm{Ne}$ II $]$ & 0.77 & $<1 \times 10^{-6}$ & 0.61 & $<1 \times 10^{-6}$ & 0.49 & 0.08 & $<1 \times 10^{-6}$ \\
\hline$[\mathrm{Ne} \mathrm{v}]_{14 \mu \mathrm{m}}-[\mathrm{Ne} \mathrm{III}]$ & 0.86 & $<1 \times 10^{-6}$ & 0.75 & $<1 \times 10^{-6}$ & 0.67 & 0.08 & $<1 \times 10^{-6}$ \\
\hline$[\mathrm{Ne} \mathrm{v}]_{14 \mu \mathrm{m}}-[\mathrm{O}$ IV] & 0.92 & $<1 \times 10^{-6}$ & 0.78 & $<1 \times 10^{-6}$ & 0.72 & 0.08 & $<1 \times 10^{-6}$ \\
\hline$[\mathrm{Ne} \mathrm{v}]_{24 \mu \mathrm{m}}-[\mathrm{Ne} \mathrm{v}]_{14}$ & 0.92 & $<1 \times 10^{-6}$ & 0.81 & $<1 \times 10^{-6}$ & 0.77 & 0.08 & $<1 \times 10^{-6}$ \\
\hline \multicolumn{8}{|l|}{ Seyfert 2 Galaxies } \\
\hline BAT-[Ne II] & 0.63 & $<7.13 \times 10^{-5}$ & 0.48 & $<7 \times 10^{-5}$ & 0.40 & 0.11 & $<5 \times 10^{-4}$ \\
\hline BAT-[Ne III] & 0.71 & $<1 \times 10^{-6}$ & 0.55 & $<1 \times 10^{-6}$ & 0.45 & 0.10 & $<2 \times 10^{-5}$ \\
\hline BAT-[O IV $]$ & 0.56 & $<6 \times 10^{-4}$ & 0.42 & $<5 \times 10^{-4}$ & 0.33 & 0.10 & $<2 \times 10^{-3}$ \\
\hline BAT-[Ne v] & 0.65 & $<2 \times 10^{-4}$ & 0.49 & $<1 \times 10^{-6}$ & 0.38 & 0.11 & $<8 \times 10^{-4}$ \\
\hline$[\mathrm{O} / \mathrm{IV}]-[\mathrm{Ne} \mathrm{II}]$ & 0.76 & $<1 \times 10^{-6}$ & 0.59 & $<1 \times 10^{-6}$ & 0.54 & 0.09 & $<1 \times 10^{-6}$ \\
\hline [O IV $]-[\mathrm{Ne}$ III $]$ & 0.94 & $<1 \times 10^{-6}$ & 0.81 & $<1 \times 10^{-6}$ & 0.79 & 0.08 & $<1 \times 10^{-6}$ \\
\hline$[\mathrm{Ne} \mathrm{V}]_{14 \mu \mathrm{m}}-[\mathrm{Ne}$ II $]$ & 0.73 & $<4 \times 10^{-5}$ & 0.58 & $<1 \times 10^{-6}$ & 0.52 & 0.09 & $<1 \times 10^{-6}$ \\
\hline$[\mathrm{Ne} \mathrm{v}]_{14 \mu \mathrm{m}}-[\mathrm{Ne} \mathrm{III}]$ & 0.94 & $<1 \times 10^{-6}$ & 0.83 & $<1 \times 10^{-6}$ & 0.80 & 0.10 & $<1 \times 10^{-6}$ \\
\hline$[\mathrm{Ne} \mathrm{v}]_{14 \mu \mathrm{m}}-[\mathrm{O}$ IV $]$ & 0.94 & $<1 \times 10^{-6}$ & 0.80 & $<1 \times 10^{-6}$ & 0.78 & 0.09 & $<1 \times 10^{-6}$ \\
\hline$[\mathrm{Ne} \mathrm{v}]_{24 \mu \mathrm{m}}-[\mathrm{Ne} \mathrm{v}]_{14}$ & 0.92 & $<1 \times 10^{-6}$ & 0.80 & $<1 \times 10^{-6}$ & 0.78 & 0.09 & $<1 \times 10^{-6}$ \\
\hline
\end{tabular}

Notes. $\rho_{s}$ is the Spearman rank order correlation coefficient with its associated null probability, $P_{\rho} . \tau$ represents the generalized Kendall's correlation coefficient for censored data and $\tau_{p}$ is the Kendall's coefficient for partial correlation with censored data. $P_{\tau}$ and $P_{\tau}(p)$ are the null probabilities for the generalized and partial Kendall's correlation test, respectively. We also present the calculated variance, $\sigma$, for Kendall $\tau_{p}$. We have used a partial correlation test to exclude the effect of redshift (distance) in the luminosity-luminosity correlations.

the redshift where we found no correlation, with a Spearman rank, $\rho_{s}=-0.05 ; P_{\rho}=0.66$, and Kendall test, $\tau=-0.05$; $P_{\tau}=0.51$.

\subsection{Extinction in the Mid-infrared}

If wavelength-dependent extinction is important in the midinfrared, it should be evident when comparing the strengths 


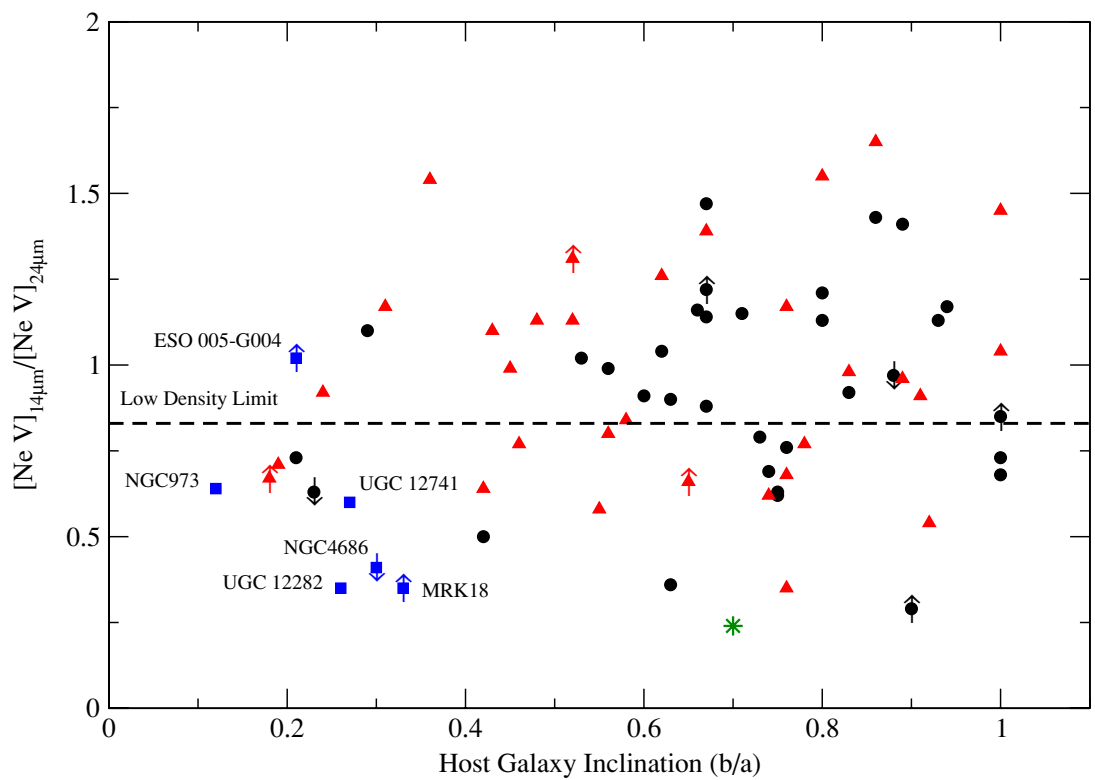

Figure 8. $[\mathrm{Ne} \mathrm{v}]_{24} \mu \mathrm{m}$ and $[\mathrm{Ne} \mathrm{v}]_{14 \mu \mathrm{m}}$ ratios as a function of host galaxy inclination for the BAT sample. Symbols are the same as in Figure 3. The horizontal line shows the low density limit. Note that five out of six of the newly detected BAT AGNs reside in inclined hosts $b / a<0.4$; hence, host galaxy extinction may be responsible for their lack of AGN signatures in the optical.

(A color version of this figure is available in the online journal.)

Table 3

Statistical Analysis Between Seyfert 1 and Seyfert 2 Galaxies

\begin{tabular}{|c|c|c|c|c|c|c|c|}
\hline \multirow[t]{2}{*}{ Parameter } & \multicolumn{3}{|c|}{ Seyfert 1} & \multicolumn{3}{|c|}{ Seyfert 2} & \multirow[b]{2}{*}{$\begin{array}{l}P_{\mathrm{K}-\mathrm{S}} \\
(\%)\end{array}$} \\
\hline & $\begin{array}{c}\text { Measurements } \\
\text { Available }\end{array}$ & Median & $\begin{array}{l}\text { Standard } \\
\text { Deviation }\end{array}$ & $\begin{array}{c}\text { Measurements } \\
\text { Available }\end{array}$ & Median & $\begin{array}{l}\text { Standard } \\
\text { Deviation }\end{array}$ & \\
\hline $\operatorname{LogBAT} /[\mathrm{O}$ IV $]$ & 38 & 2.5 & 0.5 & 33 & 2.4 & 0.5 & 2.4 \\
\hline $\operatorname{LogBAT} /[\mathrm{Ne}$ III] & 38 & 2.8 & 0.4 & 33 & 2.5 & 0.4 & 9.0 \\
\hline $\operatorname{LogBAT} /[\mathrm{Ne} \mathrm{v}]_{14.32 \mu \mathrm{m}}$ & 32 & 3.0 & 0.4 & 33 & 2.9 & 0.5 & 26.1 \\
\hline $\operatorname{LogBAT} /[\mathrm{Ne} \mathrm{v}]_{24.32 \mu \mathrm{m}}$ & 31 & 3.0 & 0.5 & 30 & 2.8 & 0.3 & 9.5 \\
\hline $\operatorname{LogBAT} /\left[\mathrm{Ne}_{\mathrm{II}}\right]$ & 38 & 2.9 & 0.4 & 33 & 2.7 & 0.5 & 2.8 \\
\hline$[\mathrm{Ne} \mathrm{III}] /[\mathrm{Ne} \mathrm{II}]$ & 38 & 1.2 & 0.1 & 33 & 1.4 & 0.1 & 54.8 \\
\hline [O IV]/[Ne II] & 38 & 1.8 & 0.4 & 33 & 1.7 & 0.4 & 84.5 \\
\hline [O IV $] /[\mathrm{Ne} \mathrm{III}]$ & 38 & 1.5 & 0.1 & 33 & 1.7 & 0.1 & 91.4 \\
\hline$[\mathrm{Ne} \mathrm{v}]_{14.32 \mu \mathrm{m} /[\mathrm{Nev}]_{24.32} \mu \mathrm{m}}$ & 29 & 1.0 & 0.3 & 30 & 1.0 & 0.4 & 94.0 \\
\hline$L_{\mathrm{BAT}}$ & 38 & 43.55 & 0.573 & 33 & 43.28 & 0.657 & 6.3 \\
\hline$L_{[\mathrm{Ne} \mathrm{II]}}$ & 38 & 40.79 & 0.613 & 33 & 40.76 & 0.799 & 82.6 \\
\hline$L_{[\mathrm{Ne} \mathrm{v}]_{14.32 \mu \mathrm{m}}}$ & 32 & 40.54 & 0.659 & 33 & 40.33 & 0.886 & 69.8 \\
\hline$\left.L_{[\mathrm{Ne}} \mathrm{III}\right]$ & 38 & 40.92 & 0.668 & 33 & 40.74 & 0.776 & 50.9 \\
\hline$L_{[\mathrm{Ne} \mathrm{v}]_{24.32 \mu \mathrm{m}}}$ & 31 & 40.52 & 0.623 & 30 & 40.44 & 0.746 & 28.6 \\
\hline$L_{[\mathrm{O} \mathrm{Iv}]}$ & 38 & 40.98 & 0.744 & 33 & 41.00 & 0.793 & 98.1 \\
\hline
\end{tabular}

Notes. The last column, $P_{\mathrm{K}-\mathrm{S}}$, represents the K-S test null probability. Upper limits for the $[\mathrm{Ne} \mathrm{v}]$ fluxes are not included. This table also includes information about the numbers of Seyfert 1 and Seyfert 2 galaxies, median values, and standard deviations of the mean for the measured quantities.

of [Ne v] $14.32 \mu \mathrm{m}$ and [Ne v] $24.32 \mu \mathrm{m}$. Dudik et al. (2007) calculated the theoretical values of this ratio as a function of density and electron temperature (see their Figure 1). As density increases, the $24.32 \mu \mathrm{m}$ line is suppressed relative to the $14.32 \mu \mathrm{m}$ line, hence, the most likely way in which the ratio can fall below the theoretical low density limit is extinction, suggesting that it should be stronger for the shorter-wavelength line. In Figure 7, we show the correlation between the two $[\mathrm{Ne} \mathrm{v}]$ lines for the BAT sample, with the theoretical low density limit over-plotted. We note that the correlation between the [Ne v] emission lines is strong and is similar in both flux and luminosities, meaning that there is a true linear correlation (see Table 4).
For the BAT sample the average $[\mathrm{Ne} \mathrm{v}]_{14.32 \mu \mathrm{m} /[\mathrm{Ne} \mathrm{v}]_{24.32} \mu \mathrm{m}}$ ratio is above the low density limit and similar for Seyfert 1 and Seyfert 2 galaxies, $1.0 \pm 0.3$ and $1.0 \pm 0.4$, respectively. The K-S test for this ratio returns a $\sim 94.0 \%$ probability of the null hypothesis, i.e., that there is no difference between Seyfert 1 and Seyfert 2 galaxies; however, Seyfert 2 galaxies show a bigger dispersion. For a gas temperature of $10^{4} \mathrm{~K}$, these results suggest an electron density of $n_{e} \approx 10^{3} \mathrm{~cm}^{-3}$, in agreement with previous studies (e.g., Sturm et al. 2002; Dudik et al. 2007; Tommasin et al. 2008). On the other hand, five out of six of the newly BAT-detected AGNs have ratios below the low density limit, with an average of $0.5 \pm 0.2$, indicating possible dust extinction. In Figure 8, we compared the $[\mathrm{Ne} \mathrm{v}]$ line ratio to the 
Table 4

Linear Regressions for the Mid-infrared and 14-195 keV Luminosities

\begin{tabular}{|c|c|c|}
\hline $\log X-\log Y$ & $\begin{array}{c}Y=a X+b \\
a\end{array}$ & $b$ \\
\hline \multicolumn{3}{|l|}{ All Sample } \\
\hline BAT-[Ne II] & $0.72 \pm 0.11$ & $9.60 \pm 4.85$ \\
\hline BAT-[Ne III] & $0.88 \pm 0.06$ & $2.58 \pm 2.70$ \\
\hline BAT-[O IV] & $0.93 \pm 0.08$ & $0.42 \pm 3.32$ \\
\hline BAT- $[\mathrm{Ne} \mathrm{v}]_{14 \mu \mathrm{m}}$ & $1.00 \pm 0.10$ & $-3.28 \pm 4.51$ \\
\hline$[\mathrm{O}$ IV $]-[\mathrm{Ne}$ II $]$ & $0.85 \pm 0.07$ & $5.83 \pm 2.67$ \\
\hline [O IV] $-[\mathrm{Ne}$ III] & $0.90 \pm 0.04$ & $3.96 \pm 1.65$ \\
\hline$[\mathrm{Ne} \mathrm{III}]-[\mathrm{Ne} \mathrm{II}]$ & $0.94 \pm 0.04$ & $2.22 \pm 1.80$ \\
\hline$[\mathrm{Ne} \mathrm{v}]_{14 \mu \mathrm{m}}-[\mathrm{Ne}$ II $]$ & $0.67 \pm 0.06$ & $13.46 \pm 2.35$ \\
\hline$[\mathrm{Ne} \mathrm{v}]_{14 \mu \mathrm{m}}-[\mathrm{Ne}$ III $]$ & $0.82 \pm 0.04$ & $7.80 \pm 1.43$ \\
\hline$[\mathrm{Ne} \mathrm{v}]_{14 \mu \mathrm{m}}-[\mathrm{O}$ IV] & $0.93 \pm 0.03$ & $3.59 \pm 1.20$ \\
\hline$[\mathrm{Ne} \mathrm{v}]_{24 \mu \mathrm{m}}-[\mathrm{Ne} \mathrm{v}]_{14 \mu \mathrm{m}}$ & $0.89 \pm 0.05$ & $4.52 \pm 2.20$ \\
\hline \multicolumn{3}{|l|}{ Seyfert 1 Galaxies } \\
\hline BAT-[Ne II] & $0.77 \pm 0.08$ & $7.08 \pm 3.69$ \\
\hline BAT-[Ne III] & $0.93 \pm 0.08$ & $0.43 \pm 3.68$ \\
\hline BAT-[O IV] & $0.93 \pm 0.11$ & $0.37 \pm 5.00$ \\
\hline BAT- $[\mathrm{Ne} \mathrm{v}]_{14 \mu \mathrm{m}}$ & $0.91 \pm 0.15$ & $0.79 \pm 6.65$ \\
\hline$[\mathrm{O}$ IV $]-[\mathrm{Ne}$ II $]$ & $0.84 \pm 0.10$ & $6.21 \pm 3.91$ \\
\hline [O IV]-[Ne III] & $0.91 \pm 0.07$ & $3.57 \pm 2.78$ \\
\hline$[\mathrm{Ne}$ III] $-[\mathrm{Ne}$ II $]$ & $0.92 \pm 0.06$ & $3.03 \pm 2.56$ \\
\hline$[\mathrm{Ne} \mathrm{V}]_{14 \mu \mathrm{m}}-[\mathrm{Ne} \mathrm{II}]$ & $0.74 \pm 0.09$ & $10.65 \pm 3.76$ \\
\hline$[\mathrm{Ne} \mathrm{v}]_{14 \mu \mathrm{m}}-[\mathrm{Ne}$ III $]$ & $0.90 \pm 0.06$ & $4.44 \pm 2.53$ \\
\hline$[\mathrm{Ne} \mathrm{v}]_{14 \mu \mathrm{m}}-[\mathrm{O}$ IV $]$ & $1.00 \pm 0.05$ & $0.62 \pm 2.16$ \\
\hline$[\mathrm{Ne} \mathrm{v}]_{24} \mu \mathrm{m}-[\mathrm{Ne} \mathrm{v}]_{14 \mu \mathrm{m}}$ & $1.02 \pm 0.05$ & $-0.69 \pm 1.50$ \\
\hline \multicolumn{3}{|l|}{ Seyfert 2 Galaxies } \\
\hline BAT-[Ne II] & $0.94 \pm 0.15$ & $0.15 \pm 6.56$ \\
\hline BAT-[Ne III] & $0.99 \pm 0.10$ & $-1.82 \pm 4.42$ \\
\hline BAT-[O IV] & $1.00 \pm 0.13$ & $-2.17 \pm 5.72$ \\
\hline BAT- $[\mathrm{Ne} \mathrm{V}]_{14 \mu \mathrm{m}}$ & $1.10 \pm 0.12$ & $-7.10 \pm 5.28$ \\
\hline$\left[\mathrm{O}_{\mathrm{IV}}\right]-[\mathrm{Ne} \mathrm{II}]$ & $0.98 \pm 0.10$ & $0.48 \pm 3.89$ \\
\hline [O IV]-[Ne III] & $0.96 \pm 0.05$ & $1.47 \pm 2.14$ \\
\hline$[\mathrm{Ne}$ III $]-[\mathrm{Ne}$ II $]$ & $1.02 \pm 0.05$ & $-1.01 \pm 2.18$ \\
\hline$[\mathrm{Ne} \mathrm{V}]_{14 \mu \mathrm{m}}-[\mathrm{Ne} \mathrm{II}]$ & $0.89 \pm 0.09$ & $4.78 \pm 3.62$ \\
\hline$[\mathrm{Ne} \mathrm{v}]_{14 \mu \mathrm{m}}-[\mathrm{Ne}$ III $]$ & $0.87 \pm 0.05$ & $5.73 \pm 2.11$ \\
\hline$[\mathrm{Ne} \mathrm{v}]_{14 \mu \mathrm{m}}-[\mathrm{O} \mathrm{IV}]$ & $0.90 \pm 0.04$ & $4.45 \pm 1.50$ \\
\hline$[\mathrm{Ne} \mathrm{v}]_{24} \mu \mathrm{m}-[\mathrm{Ne} \mathrm{v}]_{14 \mu \mathrm{m}}$ & $1.07 \pm 0.04$ & $-2.97 \pm 1.48$ \\
\hline
\end{tabular}

Notes. $\log X$ and $\log Y$ represent the independent and dependent variables, respectively. $a$ and $b$ represent the regression coefficient (slope) and regression constant (intercept), respectively. For the relationship between the $14-195 \mathrm{keV}$ and mid-infrared luminosities we used the ordinary least-square regression of the dependent variable, $Y$, against the independent variable $X, \operatorname{OLS}(Y \mid X)$. For the relationship between the mid-infrared emission line luminosities we used the OLS bisector method which treat the variables symmetrically (see Isobe et al. 1990, for a review). When censored data were present we used the EM and the Buckley-James (BJ) method, if censored data were present in both variables (e.g., the $[\mathrm{Ne} \mathrm{v}]_{14}-[\mathrm{Ne} \mathrm{v}]_{24}$ relationship) the Schmitt's binned method was used (Isobe et al. 1986; Isobe \& Feigelson 1990). The results from the EM and BJ methods agree within their estimated errors; therefore, for the sake of simplicity, we present only the values from the EM regression.

host galaxy inclination. ${ }^{15}$ All of the newly BAT-detected AGNs are found in inclined host galaxies which argue in favor of a scenario where the optical signatures of the AGNs may have been missed in this group, in part, due to extinction in the plane of the host galaxy; as suggested by Kirhakos \& Steiner (1990) and Simcoe et al. (1997) and more recently by GA09. However, one must note from Figure 8 that there is no correlation between the $[\mathrm{Ne} \mathrm{v}]$ line ratio and the value of the host galaxy inclination.

\footnotetext{
15 The values for the major and minor diameters of the host galaxy, $a$ and $b$ respectively, were taken from NED.
}

Hence, host galaxy inclination cannot be solely responsible for the observed mid-infrared extinction in our sample.

In addition to providing some insight into the nature of the newly BAT-detected AGNs, these plots show that $\sim 34 \%$ of Seyfert 1 galaxies and $\sim 37 \%$ of Seyfert 2 galaxies have [Ne v] ratios below the low density limit, in good agreement with the percentage of Seyfert galaxies that fall below the low density limit for the neon ratio from the high-resolution spectroscopy of the $12 \mu \mathrm{m}$ sample (Tommasin et al. 2010). If extinction at shorter wavelengths were responsible for the neon ratio below the low density limit, it is plausible that the tightness of the $[\mathrm{Ne}$ III $]-L_{\mathrm{BAT}}$ correlation may be due to the combined effects of mid-infrared extinction and X-ray decrement due to Compton scatter in the BAT band (e.g., Meléndez et al. 2008b). Similarly, the larger scatter for [O IV] $-L_{\mathrm{BAT}}$ may be due, in part, to the lower extinction for [O IV], compared to [Ne III]; hence, the variations in the observed X-ray, due to different column densities toward the nuclear region, become the dominant effect. One must note that the mid-infrared extinction curved by Weingartner \& Draine (2001) and Li \& Draine (2001; e.g, see their Figure 16),

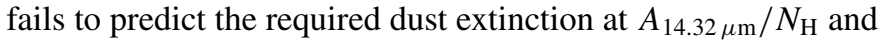

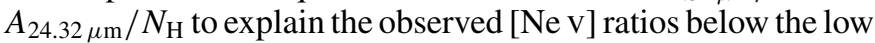
density limit. Other possibilities to explain the observed neon ratios below the low density limit must be consider, such as, aperture effects and/or adopted atomic data for neon; however, these possibilities have also failed to fully explain the observed ratios below the low density limit (e.g., Dudik et al. 2007; Tommasin et al. 2010).

\section{COMPARISON BETWEEN BAT AGNS AND OTHER SOURCES}

Following the mid-infrared properties for the BAT AGN sample we investigated the relationship between different types of galaxies. For comparison we searched the literature for the most recent Spitzer high-resolution IRS spectroscopy, from this we used the volume-limited sample of bolometrically luminous galaxies $\left(L_{\mathrm{IR}}>3 \times 10^{9} L_{\odot}\right)$ to a distance of $D<15 \mathrm{Mpc}$ of GA09 which includes HII galaxies, LINERS, optically classified AGNs and optically unclassified galaxies. We also used the recent atlas of starburst galaxies by B09 that includes starburst galaxies with and without evidence of an AGN. Finally, we used a sample of extreme starburst galaxies which includes blue compact dwarfs (BCD) from H09. When a source was present in more than one compilation we gave priority to the data analysis and extraction presented in GA09, followed by B09 and H09.

Using [ $\mathrm{Nev}$ ] $14.32 \mu \mathrm{m}$, GA09 identified AGN activity in sources that were previously not classified by their optical spectra as AGNs. While theoretical models predict that some fraction of [Ne v] can be produce by energetic starbursts (e.g., Abel \& Satyapal 2008), H09 did not detect [Ne v] emission in their sample of extreme starburst galaxies. However, some problems arise when looking at the $[\mathrm{Ne} \mathrm{v}]$ emission in AGNs. As we mentioned before, [Ne v] $14.32 \mu \mathrm{m}$ and $24.32 \mu \mathrm{m}$ were not detected in all the sources in our sample of BAT AGNs. This suggests that because of dust extinction and instrumental sensitivity, [Ne v] emission could go undetected even in intrinsically luminous AGNs. On the other hand, [Ne II], [Ne III], and [O IV] are present in AGNs, BCD, starburst, H II galaxies and have a wide range of ionization potentials and critical densities which allow us to study the connection between the active nucleus and star formation. One must note that H09 found that the most likely contributor to the [O IV] emission 


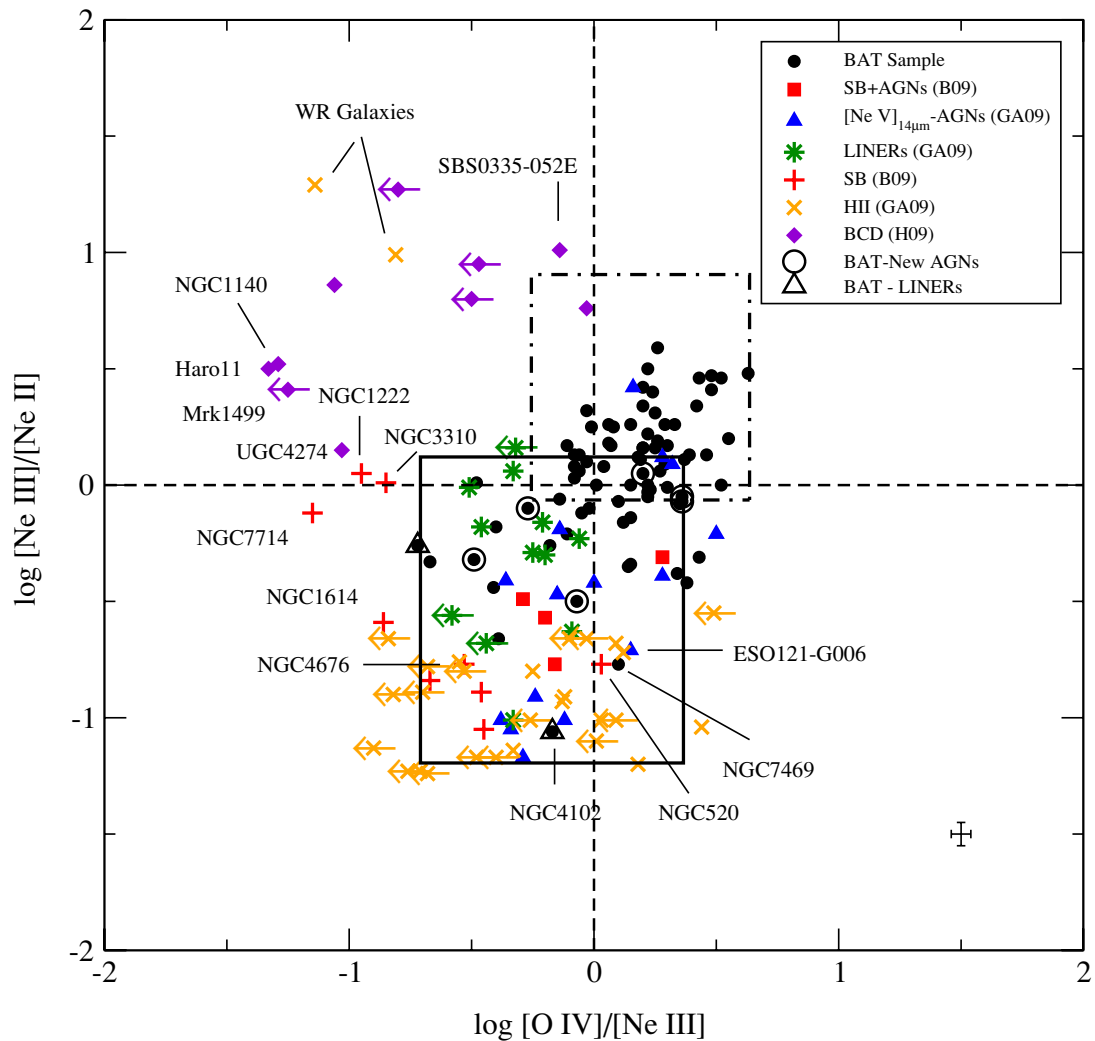

Figure 9. [Ne III]/[Ne II] vs. [O IV]/[Ne III] for a sample of different sources, including our BAT AGNs, LINERs, starburst galaxies, H II galaxies, and blue compact dwarf from high-resolution Spitzer spectra from Goulding \& Alexander (2009, GA09), Bernard-Salas et al. (2009, B09), and Hao et al. (2009, H09). Note that symbols are different than in previous figures, see legend. The dashed lines represent a value of unity for the $[\mathrm{Ne}$ III $] /[\mathrm{Ne}$ II] and $[\mathrm{O}$ IV]/[Ne III] ratios. The rectangles represent the boundaries for the sample of 74 ULIRGs (solid line) and 34 PG quasars (point-dashed line) from Veilleux et al. (2009). Interestingly, half of the BAT sample can be uniquely distinguished from SB/H II/BCD galaxies by having both $[\mathrm{Ne}$ III $] /[\mathrm{Ne} \mathrm{II}]$ and $[\mathrm{O} \mathrm{IV}] /[\mathrm{Ne}$ III] ratios greater than unity. For the ratio errors, we propagated the uncertainty for individual emission lines in quadrature. The error bars at the bottom right corner show an average error for the ratios presented in this plot.

(A color version of this figure is available in the online journal.)

in starburst and BCD galaxies is Wolf-Rayet stars. In this regard, and despite the fact that [O IV] emission traces the AGN intrinsic luminosity in pre-selected AGNs (Meléndez et al. 2008b), [O IV] cannot be solely associated with the power of the AGNs.

In Figure 9, we compare the $[\mathrm{Ne} \mathrm{III}] /[\mathrm{Ne} \mathrm{II}]$ versus [O IV]/ [Ne III] ratios. From this comparison there are four distinctive regions to note: (1) the BAT AGNs branch; (2) below the BAT AGNs are the starburst (SB) and $\mathrm{H}$ II galaxies which have an apparent [Ne II] excess; (3) above and to the left of the BAT AGNs are the BCD and two WR galaxies from GA09 (II Zw40 and NGC 1569), and (4) LINERs, which seem to follow a connecting path between AGNs and $\mathrm{SB} / \mathrm{H}$ II galaxies but are mainly found in the lower-luminosity region of the BAT AGNs branch. Despite the fact that [O IV] has been detected in BCD, $\mathrm{SB}$, and $\mathrm{H}$ II galaxies, WR and BCD galaxies cannot produce [O IV] as effectively as [Ne III], unlike in AGNs, making these sources have relative low $[\mathrm{O} \mathrm{IV}] /[\mathrm{Ne} \mathrm{III}]$ ratios. On the other hand, the hardness of the photoionizing continuum from WR stars and other energetics phenomena, such as Ultraluminous $\mathrm{X}$-ray Sources (ULXs), can ionize $\mathrm{Ne}^{+}$into $\mathrm{Ne}^{2+}$ resulting in relatively weak [Ne II] (e.g., Berghea et al. 2010), thus the high $[\mathrm{Ne}$ III $] /[\mathrm{Ne}$ II $]$ ratios observed in some $\mathrm{BCD} / \mathrm{SB}$ galaxies.

There is also a small "merge" region, at the lower ionization end of the BAT AGNs branch, that comprises LINERs and starburst galaxies with evidence for an AGN. This region exemplify the different properties of LINERs, e.g., IR-luminous and faint LINERs, with the latter having similar SED than that found in starburst galaxies (Sturm et al. 2006). Moreover, in this region we found five of the [Ne v] AGNs from GA09, some of which do not have optical signatures for an AGN. Among these [Ne v] AGNs, NGC 660 and NGC 3628 have shown some evidence to harbor a LINER (Filho et al. 2004; Dudik et al. 2005; Flohic et al. 2006). Also, based on this diagnostic, we found that NGC 520, NGC 4676, and NGC 1614 could harbor an AGN, although these starburst galaxies do not show optical evidence of such (B09). There is X-ray evidence for a Compton thick AGN in NGC 1614 (Risaliti et al. 2000a), in agreement with our diagnostic. Interestingly, these possible AGNs are found in interacting systems or mergers with strong star formation. Perhaps, interacting or merger systems may provide enough fuel to the nuclear regions of the galaxy to trigger both the nuclear star formation and the AGNs.

We also over plot in Figure 9 the sample of 74 ULIRGs and 34 Palomar-Green quasars (PG QSOs), observed with the IRS Spitzer, from Veilleux et al. (2009). From this comparison it is clear that ULIRGs show a wide range of AGNs and SB contribution to their mid-infrared emission lines, filling the gap between the BAT AGNs and the SB/H II branch and overlapping the small "merge" region defined above, in agreement with the idea that ULIRGs are composite systems mainly powered by stellar activity (Armus et al. 2007; Veilleux et al. 2009). On the other hand, PG QSOs overlap with the BAT AGN branch, in agreement with the high, typically larger than $\sim 80 \%$, AGN 
contribution to their bolometric luminosities (Veilleux et al. 2009). One must note that there are no ULIRGs detected in the Swift-BAT catalog (Tueller et al. 2010; Cusumano et al. 2010), except for the composite system NGC 6240 (Armus et al. 2006).

Moreover, most of the non-BAT AGNs presented in this work, e.g., the SB+AGN sources from B09 and [Nev] $14.32 \mu \mathrm{m}$ AGN (GA09), in other words, AGNs that have been selected because their optical and mid-infrared emission line properties have smaller mid-infrared ratios than that found for the BAT AGNs. In fact, half of the BAT sample can be uniquely distinguished from SB/H II/BCD galaxies by having both [Ne III]/ $[\mathrm{Ne} I \mathrm{II}]$ and $[\mathrm{O} \mathrm{IV}] /[\mathrm{Ne} \mathrm{III}]$ ratios greater than unity. This result suggests that the BAT sample represents a unique opportunity to study high-ionization AGNs, i.e., sources in which their optical/mid-infrared emission signatures are dominated by the AGNs. Figure 9 shows that the $[\mathrm{Ne}$ III]/[Ne II] ratio as a standalone diagnostic may overestimate the fraction of AGNs because Wolf-Rayet stars or another energetic phenomena could produce $[\mathrm{Ne} \mathrm{III}] /[\mathrm{Ne} \mathrm{II}]$ ratios even higher than those found on the BAT AGNs. However, by further constraining this ratio by using [O IV] we were able to separate AGNs from energetic starbursts and other sources.

As we mentioned before, caution must be taken when comparing fluxes between Spitzer high-resolution orders because of the differences in aperture size. However, given the similarities between the ionization potentials for [Ne III] and [O IV], it is likely that both lines originate in similar regions, thus introducing less uncertainty in the use of the $\left[\mathrm{O}_{\mathrm{IV}}\right] /[\mathrm{Ne}$ III] ratio. Nevertheless, in $\mathrm{H}$ II/SB/BCD galaxies we could be underestimating the amount of [Ne III] that is associated with the same physical conditions that produce [O IV], e.g., WR stars. This will cause this ratio, when derived from equal apertures extractions, to be displaced toward even smaller values thus, enhancing the differences between these sources and our sample of hard X-ray selected AGNs.

\section{CONCLUSIONS}

Using high-resolution Spitzer IRS spectra, we have examined the mid-infrared emission-line properties of a sample of hard X-ray selected AGNs, detected by Swift/BAT. Our principle conclusions are as follows.

1. The luminosity distribution for the mid-infrared emission lines and BAT continuum luminosities show no differences between Seyfert 1 and Seyfert 2 populations for the BAT sample. The correlations between all the midinfrared emission lines and BAT in both flux-flux and luminosity-luminosity are statistically significant, even when factoring the distance effect in luminosity-luminosity correlations. The dispersion/tightness in these correlations is due to differences in the X-ray absorbing column densities, dust extinction, and/or nuclear star formation activity. Moreover, the tight correlation found in the [Ne III]-BAT relationship suggests that, on average, there is no strong enhancement due to star formation in the [Ne III] emission in the BAT sample. Also, the slopes for the [Ne III],[Ne v], and [O IV] versus BAT luminosities relationships are smaller in Seyfert 1 galaxies than in Seyfert 2s (which are around unity), which suggests that, while the amount of extinction toward the NLR is similar in both types, the X-ray absorbing columns are large enough in Seyfert 2 s to affect the hard X-ray band, confirming the results of Meléndez et al. (2008b) and Rigby et al. (2009). This result is in agreement with the fact that the BAT/[O IV] ratio statistically separates Seyfert $1 \mathrm{~s}$ and Seyfert 2s.

2. Although all of the correlations among the mid-infrared emission lines are strong, the worst correlations are for [Ne V]-[Ne II] and [O IV]-[NeII], because of enhancement of the $[\mathrm{Ne} \mathrm{II}]$ from nuclear stellar activity (see also Meléndez et al. 2008a). While the tightness of these midinfrared correlations suggests that dust extinction is not the driving physical process behind the mid-infrared relationships, approximately $\sim 40 \%$ (including upper limits) of the sample have values for the ratio of the [Ne v] emission lines below the low-density theoretical limit, suggesting dust extinction as the physical process responsible. Exploring this, we found that all of the newly discovered BAT AGNs in our sample, which are under-luminous in [O IV] and [Ne v]14/ $24 \mu \mathrm{m}$, are found on inclined host galaxies, and all but one have $[\mathrm{Ne} \mathrm{v}]$ ratios below the critical density limit. Hence, it is likely that the newly found BAT AGNs in our sample lack optical AGN signatures because of host galaxy extinction toward their NLRs. However, the lack of correlation between host galaxy inclination and the neon ratios suggest that extinction along the plane of the host galaxy cannot be responsible for the observed extinction in all the BAT AGN samples.

3. We compared the BAT AGNs with different starburst and $\mathrm{H}$ II galaxies, so-called [Ne v] active galaxies, and LINERs (Goulding \& Alexander 2009; Bernard-Salas et al. 2009; Hao et al. 2009). We found that the BAT AGNs fall into a distinctive region based on the $[\mathrm{Ne}$ III]/[Ne II] and [O IV]/ [Ne III] ratios. Using [Ne III] and [O IV] emission, previously connected with AGN power (e.g., Gorjian et al. 2007; Meléndez et al. 2008b), does not unambiguously identify AGNs as a stand-alone diagnostic because Wolf-Rayet stars or another energetic phenomena (perhaps ULXs) could enhance the observed emission. While it is likely that detection of $[\mathrm{NeV}]$ indicates the presence of an AGN, the strongest of the [Ne v] lines have $\sim 1 / 3$ less flux than [O IV] and thus will be more difficult to detect in weak or faint AGNs. Therefore, our composite method using the [Ne II], [Ne III], and [O IV] represents a strong and simple diagnostic by using only three emission lines to identify an AGN. Based on this, we found that NGC 520, NGC 4676, and NGC 1614 could harbor an AGN, although these starburst galaxies do not show optical evidence of such (B09). There is X-ray evidence for a Compton thick AGN in NGC 1614 (Risaliti et al. 2000a), in agreement with our diagnostic. Such line diagnostic will be particularly useful to analyze spectra from new IR missions, such as the James Webb Space Telescope (Gardner et al. 2006).

We also found that ULIRGs and PG QSOs occupy two distinctive regions in our emission line diagnostic. Most ULIRGs fall into the gap between the BAT AGNs and the SB/H II branch, in agreement with the idea that ULIRGs are composite systems mainly powered by stellar activity (Armus et al. 2007; Veilleux et al. 2009). On the other hand, PG QSOs overlap with the BAT AGN branch, in agreement with the high, typically larger than $\sim 80 \%$, AGN contribution to their bolometric luminosities (Veilleux et al. 2009).

Finally, most of the non-BAT AGNs presented in our study, AGNs that have been selected because their optical and midinfrared emission line properties, have smaller mid-infrared ratios than that found for the BAT AGNs. In this regard, half of the BAT sample can be uniquely distinguished from SB/H II/ 
BCD galaxies by having both [Ne III]/[Ne II] and [O IV]/[Ne III] ratios greater than unity. Moreover, when comparing the $12 \mu \mathrm{m}$ and our BAT selected AGNs we found that the [Ne III]/[Ne II] ratio distribution between the samples is statistically different with sources in the $12 \mu \mathrm{m}$ sample having on average lower ratios than that found in the BAT AGNs, or alternatively higher recent stellar activity. This mild contamination due to star formation becomes noticeable when comparing the $12 \mu \mathrm{m}$ sample with the 14-195 keV sample, the latter of which is less biased toward star-forming systems. Despite the fact that both samples have a strong AGN contribution to their observed narrow line emission, this result suggests that the BAT sample represents a unique opportunity to study high-ionization AGNs, sources in which their optical/mid-infrared emission signatures are dominated by the AGNs, thus, providing the most representative sample in terms of galaxy population and stellar content.

We thank the referee for very useful comments that improved the paper. We also acknowledge S. Veilleux for valuable comments on the work. This research was supported by an appointment to the NASA Postdoctoral Program at the Goddard Space Flight Center, administered by Oak Ridge Associated Universities through a contract with NASA. L.M.W. acknowledges support under Hubble Fellowship program number HST-HF51263.01-A from the Space Telescope Science Institute, which is operated by the Association of Universities for Research in Astronomy, Inc., under NASA contract NAS5-26555. This research has made use of the NASA/IPAC Extragalactic Database (NED) which is operated by the Jet Propulsion Laboratory, California Institute of Technology, under contract with the National Aeronautics and Space Administration. The IRS was a collaborative venture between Cornell University and Ball Aerospace Corporation funded by NASA through the Jet Propulsion Laboratory and Ames Research Center. SMART was developed by the IRS Team at Cornell University and is available through the Spitzer Science Center at Caltech.

\section{REFERENCES}

Abel, N. P., \& Satyapal, S. 2008, ApJ, 678, 686

Akritas, M. G., \& Siebert, J. 1996, MNRAS, 278, 919

Armus, L., et al. 2006, ApJ, 640, 204

Armus, L., et al. 2007, ApJ, 656, 148

Barger, A. J., Cowie, L. L., Mushotzky, R. F., Yang, Y., Wang, W., Steffen, A. T., \& Capak, P. 2005, AJ, 129, 578

Barmby, P., et al. 2006, ApJ, 642, 126

Bassani, L., Dadina, M., Maiolino, R., Salvati, M., Risaliti, G., della Ceca, R., Matt, G., \& Zamorani, G. 1999, ApJS, 121, 473

Baum, S. A., et al. 2010, ApJ, 710, 289

Berghea, C. T., Dudik, R. P., Weaver, K. A., \& Kallman, T. R. 2010, ApJ, 708, 354

Bernard-Salas, J., et al. 2009, ApJS, 184, 230

Bevington, P. R., \& Robinson, D. K. 2003, in Data Reduction and Error Analysis for the Physical Sciences, ed. P. R. Bevington \& D. K. Robinson (Boston, MA: McGraw-Hill)

Cusumano, G., et al. 2010, A\&A, 510, A48

Dale, D. A., et al. 2006, ApJ, 646, 161

Diamond-Stanic, A. M., Rieke, G. H., \& Rigby, J. R. 2009, ApJ, 698, 623

Dudik, R. P., Satyapal, S., Gliozzi, M., \& Sambruna, R. M. 2005, ApJ, 620, 113

Dudik, R. P., Satyapal, S., \& Marcu, D. 2009, ApJ, 691, 1501

Dudik, R. P., Weingartner, J. C., Satyapal, S., Fischer, J., Dudley, C. C., \& O’Halloran, B. 2007, ApJ, 664, 71

Farrah, D., et al. 2007, ApJ, 667, 149

Filho, M. E., Fraternali, F., Markoff, S., Nagar, N. M., Barthel, P. D., Ho, L. C. \& Yuan, F. 2004, A\&A, 418, 429

Flohic, H. M. L. G., Eracleous, M., Chartas, G., Shields, J. C., \& Moran, E. C. 2006, ApJ, 647, 140

Franceschini, A., et al. 2005, AJ, 129, 2074
Gardner, J. P., et al. 2006, Space Sci. Rev., 123, 485

Genzel, R., et al. 1998, ApJ, 498, 579

George, I. M., Turner, T. J., Netzer, H., Nandra, K., Mushotzky, R. F., \& Yaqoob, T. 1998, ApJS, 114, 73

Gilli, R., Maiolino, R., Marconi, A., Risaliti, G., Dadina, M., Weaver, K. A., \& Colbert, E. J. M. 2000, A\&A, 355, 485

Gorjian, V., Cleary, K., Werner, M. W., \& Lawrence, C. R. 2007, ApJ, 655, L73 Goulding, A. D., \& Alexander, D. M. 2009, MNRAS, 398, 1165

Hao, L., Wu, Y., Charmandaris, V., Spoon, H. W. W., Bernard-Salas, J., Devost, D., Lebouteiller, V., \& Houck, J. R. 2009, ApJ, 704, 1159

Heckman, T. M., Kauffmann, G., Brinchmann, J., Charlot, S., Tremonti, C., \& White, S. D. M. 2004, ApJ, 613, 109

Heckman, T. M., Ptak, A., Hornschemeier, A., \& Kauffmann, G. 2005, ApJ, 634,161

Higdon, S. J. U., et al. 2004, PASP, 116, 975

Ho, L. C., \& Keto, E. 2007, ApJ, 658, 314

Houck, J. R., et al. 2004, ApJS, 154, 18

Hunt, L. K., \& Malkan, M. A. 1999, ApJ, 516, 660

Isobe, T., \& Feigelson, E. D. 1990, BAAS, 22, 917

Isobe, T., Feigelson, E. D., Akritas, M. G., \& Babu, G. J. 1990, ApJ, 364, 104

Isobe, T., Feigelson, E. D., \& Nelson, P. I. 1986, ApJ, 306, 490

Kessler, M. F., et al. 1996, A\&A, 315, L27

Khachikian, E. Y., \& Weedman, D. W. 1974, ApJ, 192, 581

Kirhakos, S. D., \& Steiner, J. E. 1990, AJ, 99, 1722

Koratkar, A., \& Blaes, O. 1999, PASP, 111,

Koss, M., et al. 2010, ApJ, in press

Kuraszkiewicz, J. K., et al. 2003, ApJ, 590, 128

La Massa, S. M., Heckman, T. M., Ptak, A., Hornschemeier, A., Martins, L., Sonnentrucker, P., \& Tremonti, C. 2009, ApJ, 705, 568

Landi, R., et al. 2007, ApJ, 669, 109

Levine, A. M., et al. 1984, ApJS, 54, 581

Li, A., \& Draine, B. T. 2001, ApJ, 554, 778

Lutz, D., Maiolino, R., Spoon, H. W. W., \& Moorwood, A. F. M. 2004, A\&A, 418, 465

Lutz, D., Yan, L., Armus, L., Helou, G., Tacconi, L. J., Genzel, R., \& Baker, A. J. 2005, ApJ, 632, L13

Markwardt, C. B., Tueller, J., Skinner, G. K., Gehrels, N., Barthelmy, S. D., \& Mushotzky, R. F. 2005, ApJ, 633, L77

Masetti, N., et al. 2008, A\&A, 482, 113

Matt, G. 2000, A\&A, 355, L31

McKernan, B., Ford, K. E. S., Chang, N., \& Reynolds, C. S. 2009, MNRAS, 394, 491

Meléndez, M., Kraemer, S. B., Schmitt, H. R., Crenshaw, D. M., Deo, R. P., Mushotzky, R. F., \& Bruhweiler, F. C. 2008a, ApJ, 689, 95

Meléndez, M., et al. 2008b, ApJ, 682, 94

Mulchaey, J. S., Koratkar, A., Ward, M. J., Wilson, A. S., Whittle, M., Antonucci, R. R. J., Kinney, A. L., \& Hurt, T. 1994, ApJ, 436, 586

Mushotzky, R. 2004, in Supermassive Black Holes in the Distant Universe, ed A. J. Barger (Astrophys. Space Sci. Lib. 308; Dordrecht: Kluwer), 53

Netzer, H., Mainieri, V., Rosati, P., \& Trakhtenbrot, B. 2006, A\&A, 453, 525

Netzer, H., Shemmer, O., Maiolino, R., Oliva, E., Croom, S., Corbett, E., \& di Fabrizio, L. 2004, ApJ, 614, 558

Peterson, B. M., et al. 2004, ApJ, 613, 682

Press, W. H., Teukolsky, S. A., Vetterling, W. T., \& Flannery, B. P. 1992, Numerical recipes in FORTRAN. The Art of Scientific Computing (Cambridge: Cambridge Univ. Press)

Rees, M. J. 1984, ARA\&A, 22, 471

Rigby, J. R., Diamond-Stanic, A. M., \& Aniano, G. 2009, ApJ, 700, 1878

Risaliti, G., Gilli, R., Maiolino, R., \& Salvati, M. 2000a, A\&A, 357, 13

Risaliti, G., Maiolino, R., \& Bassani, L. 2000b, A\&A, 356, 33

Satyapal, S., Böker, T., Mcalpine, W., Gliozzi, M., Abel, N. P., \& Heckman, T. 2009, ApJ, 704, 439

Satyapal, S., Sambruna, R. M., \& Dudik, R. P. 2004, A\&A, 414, 825

Satyapal, S., Vega, D., Heckman, T., O’Halloran, B., \& Dudik, R. 2007, ApJ, 663, L9

Schweitzer, M., et al. 2006, ApJ, 649, 79

Simcoe, R., McLeod, K. K., Schachter, J., \& Elvis, M. 1997, ApJ, 489, 615

Sturm, E., Lutz, D., Verma, A., Netzer, H., Sternberg, A., Moorwood, A. F. M., Oliva, E., \& Genzel, R. 2002, A\&A, 393, 821

Sturm, E., et al. 2006, ApJ, 653, L13

Tommasin, S., Spinoglio, L., Malkan, M. A., \& Fazio, G. 2010, ApJ, 709, 1257

Tommasin, S., Spinoglio, L., Malkan, M. A., Smith, H., González-Alfonso, E., \& Charmandaris, V. 2008, ApJ, 676, 836

Tueller, J., Mushotzky, R. F., Barthelmy, S., Cannizzo, J. K., Gehrels, N., Markwardt, C. B., Skinner, G. K., \& Winter, L. M. 2008, ApJ, 681, 113 Tueller, J., et al. 2010, ApJS, 186, 378 
Tully, R. B. (ed.) 1988, Nearby Galaxies Catalog (New York: Cambridge Univ. Press)

Tully, R. B., Rizzi, L., Shaya, E. J., Courtois, H. M., Makarov, D. I., \& Jacobs, B. A. 2009 , AJ, 138, 323

Ueda, Y., et al. 2007, ApJ, 664, L79

Veilleux, S., et al. 2009, ApJS, 182, 628

Véron-Cetty, M., \& Véron, P. 2006, A\&A, 455, 773

Weedman, D. W., et al. 2005, ApJ, 633, 706

Weingartner, J. C., \& Draine, B. T. 2001, ApJ, 548, 296
Werner, M. W., et al. 2004, ApJS, 154, 1

Winter, L. M., Lewis, K. T., Koss, M., Veilleux, S., Keeney, B., \& Mushotzky, R. F. 2010, ApJ, 710, 503

Winter, L. M., Mushotzky, R. F., Reynolds, C. S., \& Tueller, J. 2009a, ApJ, 690, 1322

Winter, L. M., Mushotzky, R. F., Terashima, Y., \& Ueda, Y. 2009b, ApJ, 701, 1644

Winter, L. M., Mushotzky, R. F., Tueller, J., \& Markwardt, C. 2008, ApJ, 674, 686 